\title{
Factors that influence adherence to surgical antimicrobial prophylaxis (SAP) guidelines: a systematic review
}

\author{
Sarah Hassan $^{1^{*}}$ (D), Vincent Chan ${ }^{1}$, Julie Stevens ${ }^{1,2,3}$ and leva Stupans ${ }^{1}$
}

\begin{abstract}
Background: Despite the extensive research that has been conducted to date, practice often differs from established guidelines and will vary between individuals and organisations. It has been noted that the global uptake of local and international surgical antimicrobial prophylaxis (SAP) guidelines is poor with limited research investigating factors that affect guideline adherence. The purpose of this systematic review was to determine the reported barriers and enablers to the adherence of SAP guidelines.

Methods: A search of the literature was performed using four electronic databases (CINAHL, EMBASE, PubMed and SCOPUS) for articles published in the English language from January 1998 to December 2018. Articles were included if they were solely related to SAP and discussed the barriers or enablers to SAP guideline adherence. Articles that assessed the adherence to a range of infection control measures or discussed adherence to antibiotic treatment guidelines rather than SAP guidelines were excluded from this review. Barriers and enablers were mapped to the Theoretical Domains Framework (TDF). The Mixed Methods Appraisal Tool was used to assess the quality of included studies.
\end{abstract}

Results: A total of 1489 papers were originally retrieved, with 48 papers meeting the eligibility criteria. Barriers and enablers were mapped to 11 out of 14 TDF domains: knowledge, skills, social/professional role and identity, beliefs about capabilities, beliefs about consequences, reinforcement, memory, attention and decision processes, environmental context and resources, social influences, emotion and behavioural regulation. Barriers were further categorised into personal or organisational barriers, while enablers were arranged under commonly trialled interventions.

Conclusions: There are numerous factors that can determine the uptake of SAP guidelines. An identification and understanding of these factors at a local level is required to develop tailored interventions to enhance guideline adherence. Interventions, when used in combination, can be considered as a means of improving guideline use.

Keywords: Surgical antimicrobial prophylaxis, Guideline adherence, Personal barriers, Organisational barriers, Multifaceted interventions

\footnotetext{
* Correspondence: sarah.hassan@rmit.edu.au

'Pharmacy, School of Health and Biomedical Sciences, RMIT University, Bundoora, Victoria, Australia

Full list of author information is available at the end of the article
}

(C) The Author(s). 2021 Open Access This article is licensed under a Creative Commons Attribution 4.0 International License, which permits use, sharing, adaptation, distribution and reproduction in any medium or format, as long as you give appropriate credit to the original author(s) and the source, provide a link to the Creative Commons licence, and indicate if changes were made. The images or other third party material in this article are included in the article's Creative Commons licence, unless indicated otherwise in a credit line to the material. If material is not included in the article's Creative Commons licence and your intended use is not permitted by statutory regulation or exceeds the permitted use, you will need to obtain permission directly from the copyright holder. To view a copy of this licence, visit http://creativecommons.org/licenses/by/4.0/ The Creative Commons Public Domain Dedication waiver (http://creativecommons.org/publicdomain/zero/1.0/) applies to the data made available in this article, unless otherwise stated in a credit line to the data. 


\section{Background}

Surgical site infections (SSIs)_infections that occur at or near the site of a surgical incision within 30 days of procedure or within 90 days of prosthesis implantation-are classified as one of the most common types of nosocomial infections [1, 2], accounting for up to $38 \%$ of infections in surgical patients [3]. SSIs are often associated with a greater length of hospital stay, hospital readmissions, increased health care costs and mortality [4-6]. Surgical antimicrobial prophylaxis (SAP), the administration of antibiotics immediately prior to surgery, is a key strategy used to help prevent the development of post-operative infections, namely SSIs [7]. Whilst infection control practices such as operating room ventilation, surgical instrument sterilisation and ensuring adequate skin preparation may also play a role in preventing SSIs $[3,8,9]$, the use of SAP has been pivotal in decreasing infection rates $[10,11]$.

Studies have focused on establishing the criteria that determine the appropriateness of SAP, with recommendations being updated by key bodies such as the Centers for Disease Control and Prevention (CDC) and the World Health Organization (WHO) $[8,9,12]$. In Australia, further guidance is provided by the Australian Commission on Safety and Quality in Health Care via the Antimicrobial Stewardship Clinical Care Standards. This standard provides statements on the delivery of care to a patient with bacterial infections as well as how antibiotics should be prescribed for SAP [13].

SAP recommendations are often presented in the form of clinical practice guidelines, with suggestions on the appropriate prescribing and administration of antibiotics. Optimal SAP is dependent on fulfilment of the following key quality indicators: correct selection of antimicrobial for indication, administration of correct dose via correct route, administration of preoperative antibiotics at the correct time with intraoperative doses given at the correct interval and administration of SAP for the recommended duration [7, 14].

Clinical practice guidelines-statements that assist practitioners in their decision making and reflect the most current evidence-based research-are recommended to be used alongside a clinician's judgement in determining the best course of action for patients [15]. However, despite the evidence that is presented in such documents and the established benefits of using SAP, multiple audits have indicated that adherence rates to SAP recommendations is often suboptimal [16-19]. Studies have underlined non-concordance to many of the quality indicators, particularly a lack of adherence to timing of administration [16, 20, 21]. Extended duration of prophylaxis has also been documented and is of significant concern as it can contribute to growing antimicrobial resistance $[16-18,20]$. Poor adherence to guidelines, arising from evidence-practice gaps, can lead to suboptimal health care, increased patient harm, diminished quality of life and unnecessary costs [22].

Current recommendations listed in CDC and WHO guidelines for the prevention of SSIs advocate for no further doses to be administered once incision has been closed for most procedures, while suggesting a limited duration of use to $24 \mathrm{~h}$ for procedures where evidence is lacking $[8,9,12]$. In addition to guidance provided for appropriate SAP, the CDC and WHO guidelines also list pre-, intra- and postoperative measures for the prevention of SSIs. Key measures associated with SSI prophylaxis preoperatively include surgical hand preparation, preoperative bathing with plain or antimicrobial soap and surgical site preparation with chlorhexidine gluconate-based antiseptic solutions $[8,12]$. Maintaining normal body temperature and optimal perioperative blood glucose concentrations is recommended intraoperatively, with the use of standard wound dressings recommended over advanced dressings in the postoperative setting [9, 12].

Knowledge translation is required to ensure that science research is transferred to the clinical setting. This has been recognised as a complex and slow process, with estimates of a 17-year time lag between research and practice $[23,24]$. In addition, the translation of quality evidence-based research has been identified as challenging for health care professionals [22]. The uptake of guidelines is inconsistent across various settings [25], with its utilisation also considered slow and unpredictable [26-28]. It has been reported that guidelines are followed in $67 \%$ of decisions, although this is highly variable between physicians and guidelines [27].

Successful implementation of practice change interventions requires an understanding of the personal and organisational factors that influence behaviour. The Theoretical Domains Framework (TDF) has previously been used in health care settings to explore the determinants of guideline use $[29,30]$. TDF contains 14 domains which highlight how the interplay of individual, social and environmental factors may influence behaviour [31]. By understanding these factors through the lens of TDF, tailored interventions can be designed to address these factors and thus promote behaviour change.

Despite the knowledge of barriers and enablers to clinical guideline use in general, little is known regarding the determinants of SAP guideline uptake. Thus, the aim of this review was to identify the barriers and enablers to adherence of SAP guidelines in order to provide health care providers with a theoretically derived understanding of how to improve adherence to guidelines. The findings of this review may help improve the understanding of the personal and system-based factors that hinder the uptake of SAP guidelines, whilst also highlighting trialled 
interventions that can be employed by organisations in order to increase guideline uptake.

\section{Methods}

\section{Search strategy}

Using the Preferred Reporting Items for Systematic Reviews and Meta-Analyses (PRISMA) guidelines (Additional file 1) [32], a detailed literature search was conducted to retrieve papers that could identify the barriers and enablers to SAP guideline adherence.

Four electronic databases (CINAHL, EMBASE, PubMed and SCOPUS) were searched for articles that were published in English between January 1998 and December 2018. Search terms included "antibiotic prophylaxis", "practice guidelines" and "guideline adherence"-a combination of free text and MeSH headings were used where appropriate. The Boolean operators OR and AND were used to combine the search terms. Reference lists of full-text papers that met the eligibility criteria were also hand searched in order to identify relevant studies that may not have appeared through the database search. The search strategy for each database is listed in Additional file 2.

\section{Inclusion criteria}

Articles were included if (a) they were solely related to SAP (without the assessment of adherence to other infection control measures); (b) discussed barriers or enablers to SAP guideline adherence; (c) SAP guidelines were pre-existing before the study was conducted (either through local, institutional guidelines or through the use of national or international guidelines); (d) were in the English language; (e) were original, peer-reviewed articles; and (f) the full-text articles could be sourced.

\section{Exclusion criteria}

Articles were excluded if (a) they were not related to SAP, (b) discussed multiple infection control measures alongside SAP guideline use, (c) did not assess/discuss the factors that influence adherence to SAP guidelines, (d) discussed treatment or therapeutic doses of antibiotics rather than prophylaxis, (e) were audits that solely discussed compliance rates with SAP guidelines without an explanation of the factors that influenced use, (f) discussed perceived barriers or enablers to SAP guideline adherence (rather than reported/factual factors), (g) reviewed how guideline adherence affected infection rates, (h) discussed factors that were based on statistical analyses only and (i) SAP guidelines were developed as part of a study before assessing the effects of interventions that may influence adherence. Additionally, grey literature (e.g. conference papers and theses) as well as review papers were excluded.

\section{Selection of studies}

Database search results were exported to Endnote version X9.2 (Clarivate Analytics), where duplicates were removed. Titles and abstracts were initially screened by a single author (SH), due to the efficiency and acceptability of this process [33], and papers that were potentially relevant based on the eligibility criteria underwent a full-text review. Full-text papers were independently reviewed by two authors (SH and IS). Where discrepancies were found in the outcomes, discussions were made with two authors (VC and JS) until a consensus could be reached.

\section{Assessment of quality}

The Mixed Methods Appraisal Tool (MMAT) was used to assess the quality of included studies [34]. The MMAT is a quality appraisal tool that appraises the methodological quality of qualitative, quantitative (randomised controlled, non-randomised, quantitative descriptive) and mixed methods studies.

Ten papers (21\%) were randomly selected and independently assessed by all four authors. Discussions took place where discrepancies were identified until a consensus could be reached. The remaining papers were then independently assessed by one author $(\mathrm{SH})$, due to the acceptability of single author appraisal [33]. Papers were assigned a score based on the percentage of criteria that was met for the relevant study design (i.e. if "Yes" was selected 4 out of 5 times, a score of $80 \%$ was given). For papers that were classified as mixed methods studies, 3 sets of criteria were used to determine the final score (using the qualitative study criteria, quantitative study criteria and the mixed methods study criteria). Papers were then categorised into one of three categories-where low quality was considered to be a score between 0 and 40\%, medium quality between 41 and $70 \%$ and high quality between 71 and $100 \%$.

\section{Data extraction and analysis}

Data was extracted and tabulated. Data retrieved from the papers included a list of reported barriers that prevented adherence to guideline use as well as any enablers (i.e. interventions employed by included studies to promote adherence to SAP guidelines). For articles that described an intervention that was deemed unsuccessful, potential reasons behind the outcome were documented. Data collected on barriers and enablers were mapped to the TDF. Barriers were further categorised under the headings personal or organisational barriers, while enablers were categorised under the interventions that were employed by included studies. Mapping of domains was reviewed by all four authors and agreed upon accordingly. 


\section{Results}

\section{Article selection}

A total of 1489 studies were initially retrieved through the database search. After removing duplicated articles $(n=262)$, the title and abstracts of 1227 articles were reviewed. From this, 1132 records were excluded on the basis of title and abstract, resulting in 95 papers with potential for inclusion. The reference list of fulltext articles were also hand searched, resulting in a further 9 papers that were reviewed for eligibility. After reviewing the full-text articles, a total of 48 papers were deemed relevant and thus included for synthesis (Fig. 1). A list of articles that were excluded can be found in Additional file 3 .

\section{Characteristics of selected studies}

Of the 48 papers included in this review, the majority highlighted enablers (i.e. successful interventions) that permitted SAP guideline adherence (36/48). Six papers solely discussed barriers to guideline adherence, and three papers discussed interventions that were neither a barrier nor an enabler to guideline use while the remaining three papers highlighted factors that both prevented and promoted guideline adherence. Almost half of the studies were conducted in the USA (22/48), three in Australia, two each in Brazil, Canada, France, Italy, Jordan and the UK while the remaining studies were conducted in Belgium, China, Greece, Ireland, Korea, New Zealand, Nigeria, Qatar, South Africa, Spain and Turkey.

\section{Assessment of quality}

All 48 papers were assessed using at least one of the study designs listed in the MMAT [34]. The most common criteria used to assess the quality were questions pertinent to a non-randomised study (36/48), followed by quantitative descriptive studies (4/48) and qualitative studies (3/48). Two papers were classified as randomised control trials, two papers used two different quantitative approaches and one paper used a mixed methods study design and so was assessed accordingly.

Thirty-nine papers were deemed to be of high quality, with 25 papers scoring $80 \%$ while the remaining 14 papers received a score of $100 \%$. Of the remaining 9 papers, eight papers were classified as being of medium quality (60\% score achieved in $7 / 8$ papers, $70 \%$ for $1 / 8$ papers). Only one study was categorised as low quality, receiving a score of $40 \%$; however, we did not exclude any studies on the basis of quality. The results of individual studies can be found in Additional file 4 .

\section{Key findings of individual studies}

A summary of the findings can be found in Tables 1, 2, 3 and 4 . Table 1 highlights the reported barriers while Table 2 highlights the reported enablers of included studies. Table 3 describes studies that discussed both barriers and enablers, while Table 4 describes studies in which interventions employed were neither a barrier nor enabler to SAP guideline adherence.

\section{Barriers to guideline adherence}

A total of nine papers discussed barriers to SAP guideline adherence (Tables 1 and 3) [35-40, 77-79]. Barriers were often grouped as personal or organisational barriers and were mapped to the following nine TDF domains: knowledge [35-38, 77-79], environmental context and resources [35-40, 77-79], social/professional role and identity $[36,40,77]$, memory, attention and decision processes [36, 37], beliefs about consequences [37, 39], beliefs about capabilities [37, 38, 77, 78], emotion [37, 40], skills [37, 39, 77] and social influences [37, 40, 77].

Three papers discussed lack of guideline knowledge or awareness of guideline content as a reason behind poor SAP guideline use $[35,36,38]$. Poor communication between specialties, namely surgical and anaesthetics, also appeared as a recurrent theme in the papers $[35,36,40]$. This lack of communication often meant that tasks relating to antibiotic prescribing and administration were not delegated $[36,77]$.

\section{Enablers to guideline adherence}

A total of thirty-nine papers discussed enablers that promoted the use of SAP guidelines (Tables 2 and 3) [41-79]. Multiple interventions were developed and incorporated into practice to enhance guideline use. Eight relevant TDF domains were identified: behavioural regulation [41, 44, 45, 47, 50-60, 62-67, 69, 71, 73-76, 79], environmental context and resources [41-54, 56-60, 62-68, 70-72, 74-79], skills $[41,57,77]$, social/professional role and identity $[41,49,60$, $62,65,67,70,72,76]$, knowledge [42, 43, 45-47, 49, 50, 52, $54,56,61-64,68,70,76,78,79]$, social influences [45, 48, $49,69,78,79]$, reinforcement $[49,53,58,59,62-65,67,71$, 74] and beliefs about consequences [78]. A breakdown of the successful interventions that enabled SAP guideline use can be found in Tables 2 and 3.

\section{Interventions that had minimal effect on guideline adherence}

Three papers included interventions that failed to increase the uptake of SAP guidelines [80-82]. Of the three papers, two included educational sessions regarding SAP guidelines $[80,81]$. Preoperative checklists were also modified in an attempt to increase guideline use $[81,82]$. 


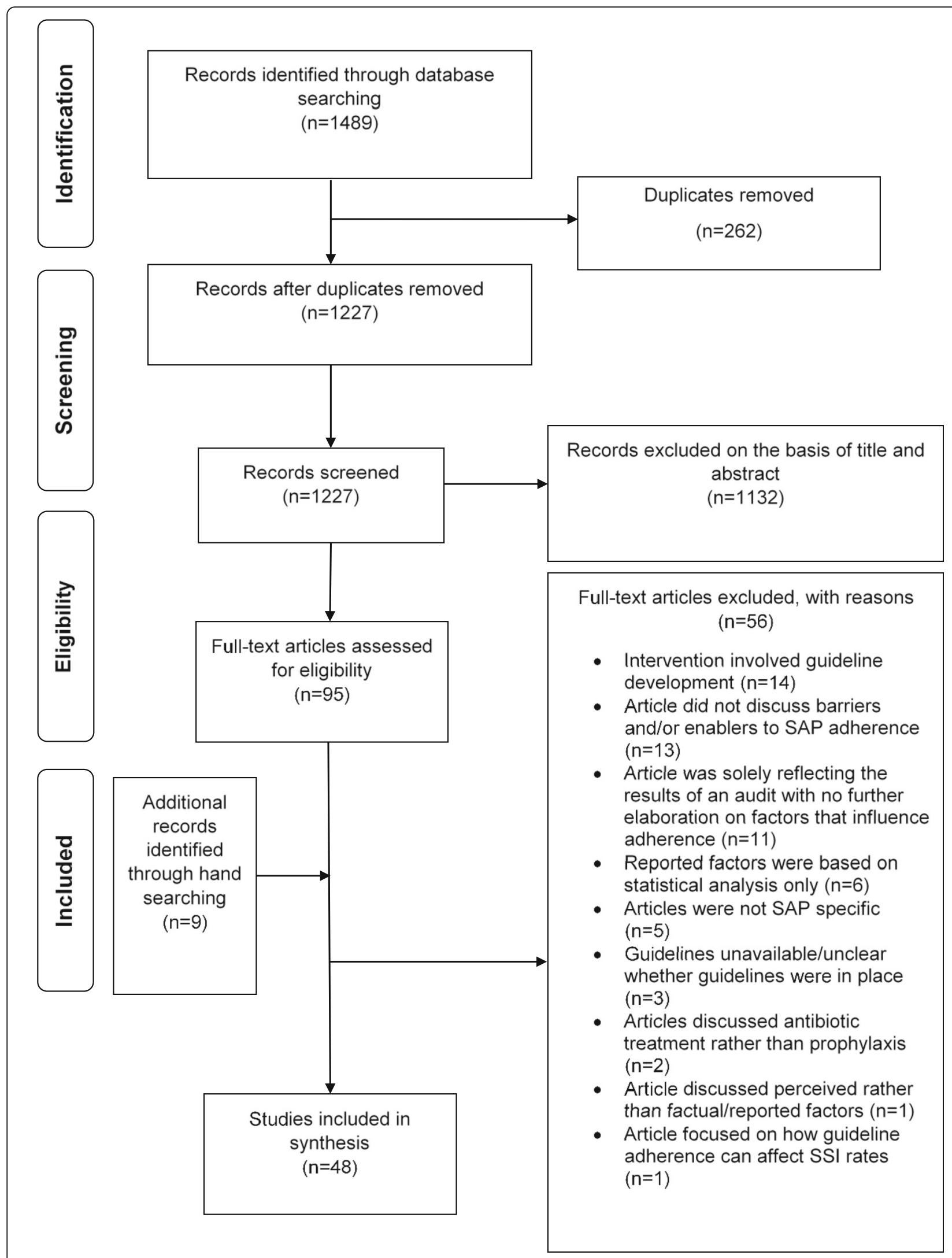

Fig 1. PRISMA flow diagram 
Table 1 Summary of reported barriers

\begin{tabular}{|c|c|c|c|}
\hline $\begin{array}{l}\text { Author (year) } \\
\text { and country }\end{array}$ & $\begin{array}{l}\text { Study design and } \\
\text { population }\end{array}$ & TDF Domain & Description of reported barrier \\
\hline \multirow{2}{*}{$\begin{array}{l}\text { Al-Azzam } \\
\text { et al. (2012) } \\
\text { [35] } \\
\text { Jordan }\end{array}$} & $\begin{array}{l}\text { Quantitative, descriptive (cross- } \\
\text { sectional survey) }\end{array}$ & Knowledge & $\begin{array}{l}\text { Personal barrier (intrapersonal): } \\
\text { - Lack of guideline knowledge }\end{array}$ \\
\hline & Physicians & $\begin{array}{l}\text { Environmental } \\
\text { context and } \\
\text { resources }\end{array}$ & $\begin{array}{l}\text { Organisational barriers: } \\
\text { - Work flow } \\
\text { - Lack of organisational communication } \\
\text { - Drug unavailability } \\
\text { - Drug cost } \\
\text { - Presence of institutional policy (preventing the use of international } \\
\text { guidelines - note that authors were determining compliance to } \\
\text { international guidelines in this paper) }\end{array}$ \\
\hline \multirow{4}{*}{$\begin{array}{l}\text { Bonfait et al. } \\
\text { (2010) [36] } \\
\text { France }\end{array}$} & Quantitative, descriptive & $\begin{array}{l}\text { Social/Professional } \\
\text { role and identity }\end{array}$ & $\begin{array}{l}\text { Personal barriers (interpersonal): } \\
\text { - Lack of role delegation for prescribing and administering antibiotics }\end{array}$ \\
\hline & \multirow[t]{3}{*}{ Orthopaedic surgeons } & Knowledge & \multirow{2}{*}{$\begin{array}{l}\text { Personal barriers (intrapersonal): } \\
\text { - Lack of awareness of guideline content } \\
\text { - Antibiotics not administered due to "negligence or oversight" }\end{array}$} \\
\hline & & $\begin{array}{l}\text { Memory, attention } \\
\text { and decision } \\
\text { processes }\end{array}$ & \\
\hline & & $\begin{array}{l}\text { Environmental } \\
\text { context and } \\
\text { resources }\end{array}$ & $\begin{array}{l}\text { Organisational barriers: } \\
\text { - Lack of communication between specialties (anaesthetists and surgeons) } \\
\text { at induction } \\
\text { - Insufficient staff training } \\
\text { - Excessive workload and inappropriate work allocation } \\
\text { - Lack of written guidelines } \\
\text { - Guidelines present in the wrong place - inaccessible in theatre or on the } \\
\text { wards }\end{array}$ \\
\hline \multirow[t]{8}{*}{$\begin{array}{l}\text { Broom et al. } \\
\text { (2018) [37] } \\
\text { Australia }\end{array}$} & Qualitative & $\begin{array}{l}\text { Memory attention } \\
\text { and decision } \\
\text { processes }\end{array}$ & \multirow{6}{*}{$\begin{array}{l}\text { Personal barriers (intrapersonal): } \\
\text {-Forgetfulness } \\
\text { - Lack of confidence in ability to protect against adverse consequences/ } \\
\text { fear of repercussions (infections) hence extended duration of prophylaxis } \\
\text { ("peace of mind") } \\
\text { - Level of experience (junior vs senior) }\end{array}$} \\
\hline & \multirow[t]{7}{*}{$\begin{array}{l}\text { Surgeons } \\
\text { Anaesthetists }\end{array}$} & $\begin{array}{l}\text { Beliefs about } \\
\text { consequences }\end{array}$ & \\
\hline & & $\begin{array}{l}\text { Beliefs about } \\
\text { capabilities }\end{array}$ & \\
\hline & & Emotion & \\
\hline & & Skills & \\
\hline & & Knowledge & \\
\hline & & Social influences & Organisational barriers: \\
\hline & & $\begin{array}{l}\text { Environmental } \\
\text { context and } \\
\text { resources }\end{array}$ & $\begin{array}{l}\text { - Culture of improvisation as the norm rather than guideline adherence } \\
\text { - Antibiotic prophylaxis is seen as low priority by staff in theatre especially if } \\
\text { competing demands are present }\end{array}$ \\
\hline \multirow{3}{*}{$\begin{array}{l}\text { Chen et al. } \\
(2018)[38] \\
\text { USA }\end{array}$} & \multirow[t]{2}{*}{ Quantitative descriptive } & Knowledge & \multirow{2}{*}{$\begin{array}{l}\text { Personal barriers (intrapersonal): } \\
\text { - Lack of awareness } \\
\text { - Reliance on personal experience to determine practice }\end{array}$} \\
\hline & & $\begin{array}{l}\text { Beliefs about } \\
\text { capabilities }\end{array}$ & \\
\hline & Paediatric electrophysiologists & $\begin{array}{l}\text { Environmental context } \\
\text { and resources }\end{array}$ & $\begin{array}{l}\text { Organisational barriers: } \\
\text { - Presence of institutional guidelines (preventing national guidelines from } \\
\text { being used - note that authors were reviewing compliance to national } \\
\text { guidelines in this study) } \\
\text { - Lack of data present for paediatric population (hence national guidelines } \\
\text { not being adhered to) }\end{array}$ \\
\hline \multirow{3}{*}{$\begin{array}{l}\text { Madubueze } \\
\text { et al. (2015) } \\
\text { [39] } \\
\text { Nigeria }\end{array}$} & Quantitative descriptive & Skills & \multirow{2}{*}{$\begin{array}{l}\text { Personal barriers (intrapersonal): } \\
\text { - Habits that have been picked up during training or practice } \\
\text { - Belief that proper aseptic techniques are not being followed on site }\end{array}$} \\
\hline & \multirow[t]{2}{*}{ Orthopaedic surgeons } & $\begin{array}{l}\text { Beliefs about } \\
\text { consequences }\end{array}$ & \\
\hline & & $\begin{array}{l}\text { Environmental } \\
\text { context and } \\
\text { resources }\end{array}$ & $\begin{array}{l}\text { Organisational barriers: } \\
\text { - Work environment sterility (not considered clean enough hence the } \\
\text { extension of antibiotic use) }\end{array}$ \\
\hline
\end{tabular}


Table 1 Summary of reported barriers (Continued)

\begin{tabular}{|c|c|c|c|}
\hline $\begin{array}{l}\text { Author (year) } \\
\text { and country }\end{array}$ & $\begin{array}{l}\text { Study design and } \\
\text { population }\end{array}$ & TDF Domain & Description of reported barrier \\
\hline \multirow{4}{*}{$\begin{array}{l}\text { Tan et al. } \\
\text { (2006) [40] } \\
\text { Canada }\end{array}$} & Qualitative & $\begin{array}{l}\text { Social/professional role } \\
\text { and identity }\end{array}$ & \multirow{3}{*}{$\begin{array}{l}\text { Personal barriers (interpersonal): } \\
\text { Role perception: } \\
\text { - Shared responsibility: belief that there is a shared responsibility in } \\
\text { administering antibiotics (should be administered by whoever it is most } \\
\text { convenient for at the time) } \\
\text { - Individual responsibility: belief that antibiotic should be administered by } \\
\text { nurse or anaesthesiologist } \\
\text { - Resignation: anaesthesiologists expressed resentment at having to } \\
\text { administer antibiotics - was considered external to scope of practice; } \\
\text { violation of medical hierarchy }\end{array}$} \\
\hline & \multirow{3}{*}{$\begin{array}{l}\text { Anaesthesiologists } \\
\text { Surgeons } \\
\text { Peri-operative administrators } \\
\text { (nurse/anaesthesia } \\
\text { administrators) } \\
\text { Nurses } \\
\text { Pharmacist }\end{array}$} & Social influences & \\
\hline & & Emotion & \\
\hline & & $\begin{array}{l}\text { Environmental } \\
\text { context and } \\
\text { resources }\end{array}$ & $\begin{array}{l}\text { Organisational barriers: } \\
\text { - Inherent unpredictability of workflow systems as well as unanticipated } \\
\text { changes to workflow } \\
\text { - Antibiotic prophylaxis considered as low priority given other competing } \\
\text { concerns in theatre } \\
\text { - Administration is seen as inconvenient as it disrupts preoperative routine } \\
\text { - Lack of verbal communication regarding antibiotics information }\end{array}$ \\
\hline
\end{tabular}

\section{Discussion}

SAP plays a key role in reducing the rates of SSIs, particularly when used alongside infection control measures such as good surgical technique, the use of hand hygiene products and ensuring patient skin preparation prior to procedure $[8,9,83]$. The appropriate use of clinical practice guidelines in a surgical setting can also contribute to good clinical practice and result in better health outcomes for the patient, whilst reducing exposure to unnecessary interventions [84]. Improving guideline uptake, particularly in regard to ensuring antibiotics are prescribed and administered only for the recommended duration, can help reduce the risk of antimicrobial resistance [9]. However, poor adherence rates to SAP guidelines has been noted [16-19]; thus, the need to determine the barriers and enablers to SAP guideline use.

The TDF has been used to explore factors relating to guideline adherence in a recent qualitative study published by Ierano et al. [85] Themes were mapped to ten domains of the TDF, including knowledge, environmental context and resources, behavioural regulation and emotion [85]. Ierano et al. [85] noted that whilst guidelines were deemed to be of value, practice may differ to guideline recommendations due to a clinician's perception of gaps in the current evidence, thus resulting in deviations from guidelines. Furthermore, although participants in that study were acutely aware of both local and national guidelines, prescriber autonomy was considered to be of greater importance [85].

We were able to map our findings to 11 TDF domains: knowledge, skills, social/professional role and identity, beliefs about capabilities, beliefs about consequences, reinforcement, memory, attention and decision processes, environmental context and resources, social influences, emotion and behavioural regulation. Barriers were further arranged under personal or organisational barriers, while enablers were further categorised under commonly trialled interventions. We present our findings in light of the TDF domains, highlighting the personal and organisational barriers as well as the interventions that can, and has, enabled the uptake of guidelines.

\section{Knowledge and environmental context and resources}

A lack of knowledge and awareness of guideline content was a frequently mentioned barrier to the adherence of SAP guidelines. Being unaware of current knowledge can result in a patient being managed with information that is no longer relevant, potentially causing patient harm [22]. It is crucial that staff undertake regular educational activities in order to assist in implementation of current evidence-based research. Educational sessions provided to clinical staff, consisting of lectures or workshops, were a popular strategy employed by many studies in an attempt to increase staff knowledge and awareness of guideline content $[46,47,49,50,52,54,56$, $61-64,68,70,76,79]$. These sessions often involved a review of the importance of SAP, its optimal prescribing and infection prevention. Whilst education plays an important role in transferring knowledge and changing practice, an effective method must be used in order for the information to be retained and acted upon. Effective methods of learning that are most successful ensure that health professionals are actively engaged in the presented content [86]. Interestingly, of the three papers that reported interventions that had minimal effect on guideline uptake, two included a form of education as an intervention [80, 81]. The poor outcome presented in these situations could be a result of the non-specific educational intervention used as well as the passive method used to disseminate information, thus 
Table 2 Summary of reported enablers

\begin{tabular}{|c|c|c|c|}
\hline $\begin{array}{l}\text { Author } \\
\text { (year) and } \\
\text { country }\end{array}$ & Study design and population & TDF Domain & Description of reported enablers \\
\hline \multirow[t]{4}{*}{$\begin{array}{l}\text { Brink et al. } \\
\text { (2017) [41] } \\
\text { South Africa }\end{array}$} & Quasi-experimental (pre-post) & $\begin{array}{l}\text { Behavioural } \\
\text { regulation }\end{array}$ & $\begin{array}{l}\text { Audit and feedback: } \\
\text { - Employment of pharmacists to implement an audit and } \\
\text { feedback model in relation to adhering to guidelines; } \\
\text { providing feedback in written and verbal form } \\
\text { - Benchmarking between hospitals and regions via } \\
\text { comparative tables and graphs to compare and contrast } \\
\text { results }\end{array}$ \\
\hline & \multirow{3}{*}{$\begin{array}{l}\text { Pharmacists } \\
\text { Surgeons } \\
\text { Anaesthetists } \\
\text { Theatre and surgical ward nurses } \\
\text { Hospital, pharmacy, nursing and theatre } \\
\text { managers }\end{array}$} & $\begin{array}{l}\text { Environmental } \\
\text { context and } \\
\text { resources }\end{array}$ & \multirow[t]{2}{*}{$\begin{array}{l}\text { Effective communication: } \\
\text { - Ensuring appropriate communication between } \\
\text { multidisciplinary parties }\end{array}$} \\
\hline & & Skills & \\
\hline & & $\begin{array}{l}\text { Social/ } \\
\text { professional } \\
\text { role and } \\
\text { identity }\end{array}$ & $\begin{array}{l}\text { Role delegation: } \\
\text { - Anaesthetists taking responsibility for antibiotic } \\
\text { administration }\end{array}$ \\
\hline \multirow{2}{*}{$\begin{array}{l}\text { Bryson et al. } \\
\text { (2015) [42] } \\
\text { UK }\end{array}$} & Quasi-experimental (pre-post) & Knowledge & \multirow{2}{*}{$\begin{array}{l}\text { Guideline dissemination: } \\
\text { - Dissemination of information through the use of advertising } \\
\text { and educational programs such as announcements on the } \\
\text { intranet, displaying posters in theatre, sending out } \\
\text { information via email as well as verbally }\end{array}$} \\
\hline & $\begin{array}{l}\text { Orthopaedic surgeons } \\
\text { Anaesthetists }\end{array}$ & $\begin{array}{l}\text { Environmental } \\
\text { context and } \\
\text { resources }\end{array}$ & \\
\hline \multirow{2}{*}{$\begin{array}{l}\text { Cameron } \\
\text { et al. (2015) } \\
\text { [43] } \\
\text { UK }\end{array}$} & $\begin{array}{l}\text { Quasi-experimental (pre-post), Quantitative } \\
\text { descriptive }\end{array}$ & Knowledge & \multirow{2}{*}{$\begin{array}{l}\text { Guideline dissemination: } \\
\text { - Guideline availability in theatre to help with decision making } \\
\text { - Guidelines presented in a simple 'traffic light system' format }\end{array}$} \\
\hline & $\begin{array}{l}\text { Consultant general surgeons } \\
\text { Anaesthetists }\end{array}$ & $\begin{array}{l}\text { Environmental } \\
\text { context and resources }\end{array}$ & \\
\hline \multirow{2}{*}{$\begin{array}{l}\text { Carlès et al. } \\
\text { (2006) [44] } \\
\text { France }\end{array}$} & Quasi-experimental (pre-post) & $\begin{array}{l}\text { Behavioural } \\
\text { regulation }\end{array}$ & \multirow{2}{*}{$\begin{array}{l}\text { Support tools: } \\
\text { - Use of personalised SAP kits for patients undergoing surgery } \\
\text { that are prepared by pharmacy in advance }\end{array}$} \\
\hline & Anaesthesiologists & $\begin{array}{l}\text { Environmental } \\
\text { context and } \\
\text { resources }\end{array}$ & \\
\hline \multirow[t]{4}{*}{$\begin{array}{l}\text { Caruso et al. } \\
\text { (2017) [45] } \\
\text { USA }\end{array}$} & Quasi-experimental (pre-post) & $\begin{array}{l}\text { Social } \\
\text { influences }\end{array}$ & $\begin{array}{l}\text { Multidisciplinary collaboration: } \\
\text { - Teamwork between specialties to develop plans in order to } \\
\text { increase SAP adherence }\end{array}$ \\
\hline & \multirow{3}{*}{$\begin{array}{l}\text { Paediatric anaesthesiologists } \\
\text { Surgeons } \\
\text { Infectious disease physicians } \\
\text { Pharmacists } \\
\text { Quality improvement specialists }\end{array}$} & $\begin{array}{l}\text { Behavioural } \\
\text { regulation }\end{array}$ & \multirow{3}{*}{$\begin{array}{l}\text { Audit and feedback: } \\
\text { - Monitoring of adherence via compliance reports } \\
\text { Support tools: } \\
\text { - Incorporating antibiotic order set into pre-existing pre-surgical } \\
\text { admission routine work } \\
\text { Guideline dissemination: } \\
\text { - Dissemination of dosage information via signs posted in } \\
\text { theatre and visual aid pocket cards }\end{array}$} \\
\hline & & $\begin{array}{l}\text { Environmental } \\
\text { context and resources }\end{array}$ & \\
\hline & & Knowledge & \\
\hline \multirow[t]{2}{*}{$\begin{array}{l}\text { Collier et al. } \\
\text { (1998) [46] } \\
\text { USA }\end{array}$} & Quasi-experimental (pre-post) & Knowledge & $\begin{array}{l}\text { Educational services: } \\
\text { - Workshops to inform clinical staff of organisational changes } \\
\text { that would be made to improve practice }\end{array}$ \\
\hline & $\begin{array}{l}\text { Vascular surgeons } \\
\text { Anaesthetists } \\
\text { Theatre and ward nurses } \\
\text { Pharmacists }\end{array}$ & $\begin{array}{l}\text { Environmental } \\
\text { context and resources }\end{array}$ & $\begin{array}{l}\text { Other enablers: } \\
\text { - Rectifying issues as they are identified }\end{array}$ \\
\hline \multirow{3}{*}{$\begin{array}{l}\text { Conaty et al. } \\
\text { (2018) [47] } \\
\text { Ireland }\end{array}$} & Quasi-experimental (pre-post) & $\begin{array}{l}\text { Behavioural } \\
\text { regulation }\end{array}$ & $\begin{array}{l}\text { Audit and feedback: } \\
\text { - Weekly audit and feedback sessions (regular surveillance) }\end{array}$ \\
\hline & \multirow{2}{*}{$\begin{array}{l}\text { Orthopaedic surgeons } \\
\text { Anaesthetists } \\
\text { Nurses } \\
\text { Pharmacists }\end{array}$} & Knowledge & \multirow{2}{*}{$\begin{array}{l}\text { Educational services: } \\
\text { - Educational workshops and reminders on appropriate SAP } \\
\text { Guideline dissemination: } \\
\text { - Dissemination of guidelines electronically and through posters } \\
\text { displayed in theatre }\end{array}$} \\
\hline & & $\begin{array}{l}\text { Environmental } \\
\text { context and resources }\end{array}$ & \\
\hline $\begin{array}{l}\text { De Almeida } \\
\text { et al. (2012) } \\
\text { [48] } \\
\text { Brazil }\end{array}$ & Quasi-experimental (pre-post) & Social influences & $\begin{array}{l}\text { Multidisciplinary collaboration: } \\
\text { - Collaboration between an ICU pharmacist and infectious } \\
\text { diseases physician when reviewing patients to ensure } \\
\text { quidelines are adhered to }\end{array}$ \\
\hline
\end{tabular}


Table 2 Summary of reported enablers (Continued)

\begin{tabular}{|c|c|c|c|}
\hline $\begin{array}{l}\text { Author } \\
\text { (year) and } \\
\text { country }\end{array}$ & Study design and population & TDF Domain & Description of reported enablers \\
\hline & $\begin{array}{l}\text { Infectious disease physician } \\
\text { Pharmacist } \\
\text { Intensivist }\end{array}$ & $\begin{array}{l}\text { Environmental } \\
\text { context and resources }\end{array}$ & $\begin{array}{l}\text { Audit and feedback: } \\
\text { - Continual surveillance through compliance reports } \\
\text { disseminated electronically and verbally }\end{array}$ \\
\hline \multirow{5}{*}{$\begin{array}{l}\text { Dimopoulou } \\
\text { et al. (2016) } \\
\text { [49] } \\
\text { Greece }\end{array}$} & Quasi-experimental (pre-post) & Knowledge & \multirow{2}{*}{$\begin{array}{l}\text { Educational services: } \\
\text { - Workshops regarding appropriate SAP and SSI prevention } \\
\text { Guideline dissemination: } \\
\text { - Distribution of guidelines to clinical team }\end{array}$} \\
\hline & \multirow[t]{4}{*}{$\begin{array}{l}\text { Paediatric surgeons, nurses and } \\
\text { anaesthetists }\end{array}$} & $\begin{array}{l}\text { Environmental } \\
\text { context and resources }\end{array}$ & \\
\hline & & $\begin{array}{l}\text { Social/professional } \\
\text { role and identity }\end{array}$ & $\begin{array}{l}\text { Role delegation: } \\
\text { - Transferring the responsibility of SAP prescribing to the } \\
\text { surgeon, whilst administration of antibiotics is to be carried out } \\
\text { by the ward nurse or anaesthetist }\end{array}$ \\
\hline & & Reinforcement & Local opinion leader: \\
\hline & & Social influences & $\begin{array}{l}\text { - Use of a champion or a leader to assist with adhering to } \\
\text { guidelines (to reinforce guideline use) }\end{array}$ \\
\hline \multirow{3}{*}{$\begin{array}{l}\text { Garcell et al. } \\
\text { (2017) [50] } \\
\text { Qatar }\end{array}$} & Quasi-experimental (pre-post) & $\begin{array}{l}\text { Behavioural } \\
\text { regulation }\end{array}$ & $\begin{array}{l}\text { Audit and feedback: } \\
\text { - Regular audit and feedback sessions }\end{array}$ \\
\hline & \multirow[t]{2}{*}{ Surgical staff } & Knowledge & \multirow{2}{*}{$\begin{array}{l}\text { Educational services: } \\
\text { - Discussions around local SAP policy }\end{array}$} \\
\hline & & $\begin{array}{l}\text { Environmental } \\
\text { context and resources }\end{array}$ & \\
\hline \multirow{2}{*}{$\begin{array}{l}\text { Haynes et al. } \\
\text { (2011) [51] } \\
\text { USA }\end{array}$} & Quasi-experimental (pre-post) & $\begin{array}{l}\text { Behavioural } \\
\text { regulation }\end{array}$ & \multirow{2}{*}{$\begin{array}{l}\text { Support tools: } \\
\text { - Use of a special antibiotic order set with an automated time } \\
\text { system to force cessation of antibiotic use once time was } \\
\text { exceeded. Removing the prescribing duration from the } \\
\text { prescriber }\end{array}$} \\
\hline & Physicians & $\begin{array}{l}\text { Environmental } \\
\text { context and resources }\end{array}$ & \\
\hline \multirow{3}{*}{$\begin{array}{l}\text { Hermsen } \\
\text { et al. (2008) } \\
\text { [52] } \\
\text { USA }\end{array}$} & Quasi-experimental (pre-post) & $\begin{array}{l}\text { Behavioural } \\
\text { regulation }\end{array}$ & \multirow{3}{*}{$\begin{array}{l}\text { Support tools: } \\
\text { - Development and use of a standardised antibiotic order form } \\
\text { to provide guidance on antibiotic choice, duration and dose } \\
\text { Educational services: } \\
\text { - Educational sessions to doctors, pharmacists and nurses in } \\
\text { regards to the form and the Surgical Infection Prevention } \\
\text { Program }\end{array}$} \\
\hline & $\begin{array}{l}\text { Physicians } \\
\text { Pharmacists }\end{array}$ & $\begin{array}{l}\text { Environmental } \\
\text { context and resources }\end{array}$ & \\
\hline & & Knowledge & \\
\hline \multirow{3}{*}{$\begin{array}{l}\text { Hincker et al. } \\
\text { (2017) [53] } \\
\text { USA }\end{array}$} & Quasi-experimental (pre-post) & $\begin{array}{l}\text { Behavioural } \\
\text { regulation }\end{array}$ & \multirow{3}{*}{$\begin{array}{l}\text { Support tools: } \\
\text { - Modification of EMR system to include decision support tool to } \\
\text { guide antibiotic choice and re-dosing interval } \\
\text { Real-time reminders: } \\
\text { - Electronic reminder (on EMR) to indicate when patient should } \\
\text { be re-dosed; real-time information }\end{array}$} \\
\hline & \multirow[t]{2}{*}{ Anaesthesia staff } & $\begin{array}{l}\text { Environmental } \\
\text { context and resources }\end{array}$ & \\
\hline & & Reinforcement & \\
\hline \multirow{3}{*}{$\begin{array}{l}\text { Kao et al. } \\
\text { (2010) [54] } \\
\text { USA }\end{array}$} & Staggered cohort & Knowledge & \multirow{3}{*}{$\begin{array}{l}\text { Educational sessions: } \\
\text { - Lectures to clinical staff } \\
\text { - Drawing attention to importance of compliance } \\
\text { Support tools: } \\
\text { - Use of a preoperative checklist and standardised forms to } \\
\text { order antibiotics } \\
\text { Other enablers: } \\
\text { - Staggering introduction of interventions to give staff an } \\
\text { opportunity to implement change }\end{array}$} \\
\hline & Anaesthesia and surgical staff & $\begin{array}{l}\text { Environmental } \\
\text { context and resources }\end{array}$ & \\
\hline & & $\begin{array}{l}\text { Behavioural } \\
\text { regulation }\end{array}$ & \\
\hline \multirow{2}{*}{$\begin{array}{l}\text { Kim et al. } \\
(2012)[55] \\
\text { Korea }\end{array}$} & Quasi-experimental (pre-post) & Behavioural & \multirow{2}{*}{$\begin{array}{l}\text { Audit and feedback: } \\
\text { - Reporting of results to the public as well as to hospitals }\end{array}$} \\
\hline & Not specified & & \\
\hline \multirow{3}{*}{$\begin{array}{l}\text { Kritchevsky } \\
\text { et al. (2008) } \\
\text { [56] } \\
\text { USA }\end{array}$} & Cluster randomised trial & $\begin{array}{l}\text { Behavioural } \\
\text { regulation }\end{array}$ & \multirow{3}{*}{$\begin{array}{l}\text { Support tools: } \\
\text {-Development of prewritten order set for antibiotics } \\
\text {-Guidelines, forms and literature reviews shared amongst } \\
\text { intervention hospitals } \\
\text {-Monthly conferences } \\
\text { Audit and feedback: } \\
\text {-Comparative feedback reports sent to all participating hospitals } \\
\text { Educational services: } \\
\text { - Monthly conferences held to discuss issues and successes } \\
\text { experienced by participating intervention hospitals } \\
\text {-Meetings held to discuss strategies on how to overcome } \\
\text { obstacles related to practice }\end{array}$} \\
\hline & $\begin{array}{l}\text { Physician assistants } \\
\text { Nurse practitioners }\end{array}$ & $\begin{array}{l}\text { Environmental } \\
\text { context and resources }\end{array}$ & \\
\hline & $\begin{array}{l}\text { Pharmacists } \\
\text { Surgeons } \\
\text { Anaesthesiologists }\end{array}$ & Knowledge & \\
\hline
\end{tabular}


Table 2 Summary of reported enablers (Continued)

\begin{tabular}{|c|c|c|c|}
\hline $\begin{array}{l}\text { Author } \\
\text { (year) and } \\
\text { country }\end{array}$ & Study design and population & TDF Domain & Description of reported enablers \\
\hline \multirow{3}{*}{$\begin{array}{l}\text { Lingard et al. } \\
\text { (2011) [57] } \\
\text { Canada }\end{array}$} & Quasi-experimental (pre-post) & $\begin{array}{l}\text { Behavioural } \\
\text { regulation }\end{array}$ & \multirow{3}{*}{$\begin{array}{l}\text { Support tools: } \\
\text { - Use of a checklist to prompt antibiotic use and documentation } \\
\text { Effective communication: } \\
\text { - Comprehensive preoperative team briefings: enhanced } \\
\text { communication between multidisciplinary staff members }\end{array}$} \\
\hline & \multirow{2}{*}{$\begin{array}{l}\text { General surgeons } \\
\text { Anaesthesiologists } \\
\text { Theatre nurses } \\
\text { Technical assistants }\end{array}$} & $\begin{array}{l}\text { Environmental } \\
\text { context and resources }\end{array}$ & \\
\hline & & Skills & \\
\hline \multirow[t]{3}{*}{$\begin{array}{l}\text { Nair et al. } \\
\text { (2010) [58] } \\
\text { USA }\end{array}$} & Quasi-experimental (pre-post) & $\begin{array}{l}\text { Environmental } \\
\text { context and resources }\end{array}$ & \multirow{3}{*}{$\begin{array}{l}\text { Support tools: } \\
\text { - Implementation of AIMS: electronic anaesthesia documentation } \\
\text { system } \\
\text { Audit and feedback: } \\
\text { - Regular performance reports indicating success rates } \\
\text { - Electronic reminders (via email) to document antibiotic } \\
\text { information if missing in AIMS } \\
\text { Real-time reminders: } \\
\text { - Real-time electronic feedback and reminders via a decision sup- } \\
\text { port system known as SAMs which is linked to AIMS; reminders } \\
\text { to carry out specific action }\end{array}$} \\
\hline & \multirow[t]{2}{*}{$\begin{array}{l}\text { Anaesthesiologists } \\
\text { Nurse anaesthetists }\end{array}$} & $\begin{array}{l}\text { Behavioural } \\
\text { regulation }\end{array}$ & \\
\hline & & Reinforcement & \\
\hline \multirow{3}{*}{$\begin{array}{l}\text { Nair et al. } \\
\text { (2011) [59] } \\
\text { USA }\end{array}$} & Quasi-experimental (pre-post) & $\begin{array}{l}\text { Environmental } \\
\text { context and resources }\end{array}$ & \multirow{3}{*}{$\begin{array}{l}\text { Real-time reminders: } \\
\text { - Use of SAMs: real time alerts to prompt antibiotic } \\
\text { administration in a timely fashion. Frequent messages until } \\
\text { action taken }\end{array}$} \\
\hline & Anaesthesia staff & $\begin{array}{l}\text { Behavioural } \\
\text { regulation }\end{array}$ & \\
\hline & & Reinforcement & \\
\hline \multirow[t]{3}{*}{$\begin{array}{l}\text { O'Reilly et al. } \\
\text { (2006) [60] } \\
\text { USA }\end{array}$} & Quasi-experimental (pre-post) & $\begin{array}{l}\text { Environmental } \\
\text { context and resources }\end{array}$ & \multirow{2}{*}{$\begin{array}{l}\text { Support tools: } \\
\text { - Modification of electronic perioperative systems to allow } \\
\text { documentation of antibiotic particulars } \\
\text { Audit and feedback: } \\
\text { - Regular performance feedback to individual staff members } \\
\text { (electronic). Verbal feedback given to staff who are constantly } \\
\text { non-compliant } \\
\text { - Publication of performance results to serve as a reminder as } \\
\text { well as verbal reminders during staff meetings }\end{array}$} \\
\hline & \multirow[t]{2}{*}{$\begin{array}{l}\text { Surgeons } \\
\text { Nurses } \\
\text { Anaesthesia staff }\end{array}$} & $\begin{array}{l}\text { Behavioural } \\
\text { regulation }\end{array}$ & \\
\hline & & $\begin{array}{l}\text { Social/professional } \\
\text { role and identity }\end{array}$ & $\begin{array}{l}\text { Role delegation: } \\
\text { - Anaesthetists responsible for administering antibiotics }\end{array}$ \\
\hline \multirow{2}{*}{$\begin{array}{l}\text { Ozgun et al. } \\
\text { (2010) [61] } \\
\text { Turkey }\end{array}$} & Quasi-experimental (pre-post) & Knowledge & \multirow[b]{2}{*}{$\begin{array}{l}\text { Educational services: } \\
\text { - Educational sessions held regarding SAP principles as well as } \\
\text { what is considered inappropriate use } \\
\text { - Data on current practice presented to staff } \\
\text { - Discussion of specialty specific issues discussed with surgical } \\
\text { team } \\
\text { Guideline dissemination: } \\
\text { - SAP guidelines distributed during staff meetings and displayed } \\
\text { throughout hospital }\end{array}$} \\
\hline & $\begin{array}{l}\text { Surgeons } \\
\text { Anaesthetists } \\
\text { Nurses }\end{array}$ & $\begin{array}{l}\text { Environmental } \\
\text { context and resources }\end{array}$ & \\
\hline \multirow{6}{*}{$\begin{array}{l}\text { Parker et al. } \\
\text { (2007) [62] } \\
\text { USA }\end{array}$} & Quasi-experimental (pre-post) & $\begin{array}{l}\text { Behavioural } \\
\text { regulation }\end{array}$ & \multirow{3}{*}{$\begin{array}{l}\text { Support tools: } \\
\text {-Use of standardised preoperative antibiotic order forms } \\
\text { Real time reminders: } \\
\text {-Use of an anaesthesia record keeping system to remind } \\
\text { anaesthesia provider of appropriate time to administer antibiotics }\end{array}$} \\
\hline & \multirow{5}{*}{$\begin{array}{l}\text { Non-cardiac surgeons } \\
\text { Anaesthesiologists and anaesthesia care } \\
\text { providers } \\
\text { Preoperative nursing staff }\end{array}$} & $\begin{array}{l}\text { Environmental } \\
\text { context and resources }\end{array}$ & \\
\hline & & Reinforcement & \\
\hline & & $\begin{array}{l}\text { Social/professional } \\
\text { role and identity }\end{array}$ & $\begin{array}{l}\text { Role delegation: } \\
\text {-Delegating role of confirming and administering antibiotic } \\
\text { prophylaxis to anaesthesia personnel } \\
\text {-Antibiotics sent with patients to theatre in order to assist with } \\
\text { its administration before incision }\end{array}$ \\
\hline & & Knowledge & \multirow{2}{*}{$\begin{array}{l}\text { Educational sessions: } \\
\text {-Educating staff on antibiotic prophylaxis in order to change } \\
\text { attitudes towards use }\end{array}$} \\
\hline & & $\begin{array}{l}\text { Environmental } \\
\text { context and resources }\end{array}$ & \\
\hline $\begin{array}{l}\text { Ribed et al. } \\
\text { (2018) [63] } \\
\text { Spain }\end{array}$ & Quasi-experimental (pre-post) & $\begin{array}{l}\text { Environmental } \\
\text { context and resources }\end{array}$ & $\begin{array}{l}\text { Support tools: } \\
\text { - Prepopulating SAP information onto the CPOE to assist with } \\
\text { correct antibiotic prescribing }\end{array}$ \\
\hline
\end{tabular}


Table 2 Summary of reported enablers (Continued)

\begin{tabular}{|c|c|c|c|}
\hline $\begin{array}{l}\text { Author } \\
\text { (year) and } \\
\text { country }\end{array}$ & Study design and population & TDF Domain & Description of reported enablers \\
\hline & $\begin{array}{l}\text { Orthopaedic surgeons } \\
\text { Nurses }\end{array}$ & $\begin{array}{l}\text { Behavioural } \\
\text { regulation }\end{array}$ & $\begin{array}{l}\text { Real-time reminders: } \\
\text { - Integrating reminders into clinician's workflow to modify }\end{array}$ \\
\hline & Pharmacists & Reinforcement & $\begin{array}{l}\text { patient's prescription based on lab results } \\
\text { Educational services: }\end{array}$ \\
\hline & & Knowledge & $\begin{array}{l}\text { - Educational sessions to increase SAP awareness } \\
\text { - Pharmacy led training sessions on how to use the CPOE }\end{array}$ \\
\hline \multirow{4}{*}{$\begin{array}{l}\text { Riggi et al. } \\
\text { (2014) [64] } \\
\text { USA }\end{array}$} & Quasi-experimental (pre-post) & $\begin{array}{l}\text { Environmental } \\
\text { context and resources }\end{array}$ & \multirow{4}{*}{$\begin{array}{l}\text { Real-time reminders: } \\
\text { - Use of an automated intraoperative paging system to ensure } \\
\text { antibiotics are re-dosed at the appropriate time during lengthy } \\
\text { procedures } \\
\text { Educational services: } \\
\text { - Education sessions to anaesthesiology and surgical staff } \\
\text { Audit and feedback: } \\
\text { - Feedback to staff when non-compliance occurred (to individual } \\
\text { staff member and chairman of anaesthesiology) } \\
\text { Other enablers: } \\
\text { - Standardisation of SAP protocol (hospital wide) }\end{array}$} \\
\hline & Surgical staff & Knowledge & \\
\hline & Anaesthesia staff & $\begin{array}{l}\text { Behavioural } \\
\text { regulation }\end{array}$ & \\
\hline & & Reinforcement & \\
\hline \multirow{5}{*}{$\begin{array}{l}\text { Ritchie et al. } \\
\text { (2004) [65] } \\
\text { New Zealand }\end{array}$} & Quasi-experimental (pre-post) & $\begin{array}{l}\text { Behavioural } \\
\text { regulation }\end{array}$ & \multirow{4}{*}{$\begin{array}{l}\text { Real-time reminders: } \\
\text { - Use of a pre-printed sticker on medication chart that included } \\
\text { antibiotic particulars (dose, duration and dosing interval) to as- } \\
\text { sist with correct prescribing: information present at point of } \\
\text { care - immediate reminder of antibiotic policy }\end{array}$} \\
\hline & Anaesthetists & & \\
\hline & & Reinforcement & \\
\hline & & $\begin{array}{l}\text { Environmental } \\
\text { context and resources }\end{array}$ & \\
\hline & & $\begin{array}{l}\text { Social/professional } \\
\text { role and identity }\end{array}$ & $\begin{array}{l}\text { Role delegation: } \\
\text { - Anaesthetists responsible for applying sticker as they administer } \\
\text { first dose }\end{array}$ \\
\hline \multirow{2}{*}{$\begin{array}{l}\text { Rosenberg et al. } \\
\text { (2008) [66] } \\
\text { USA }\end{array}$} & Quasi-experimental (pre-post) & $\begin{array}{l}\text { Behavioural } \\
\text { regulation }\end{array}$ & \multirow{2}{*}{$\begin{array}{l}\text { Support tools: } \\
\text { - "Piggybacking" antibiotic administration verification to surgical } \\
\text { time out sheet (preoperative checklist). Acts as a prompt to } \\
\text { ensure antibiotics are administered prior to incision }\end{array}$} \\
\hline & $\begin{array}{l}\text { Anaesthesiologists } \\
\text { Theatre nurses }\end{array}$ & $\begin{array}{l}\text { Environmental } \\
\text { context and resources }\end{array}$ & \\
\hline \multirow{4}{*}{$\begin{array}{l}\text { Schwann et al. } \\
\text { (2011) [67] } \\
\text { USA }\end{array}$} & Quasi-experimental (pre-post) & $\begin{array}{l}\text { Behavioural } \\
\text { regulation }\end{array}$ & \multirow{3}{*}{$\begin{array}{l}\text { Real-time reminders: } \\
\text { - Use of a POCEP to provide real-time notifications during a pro- } \\
\text { cedure to administer antibiotics: reminder system }\end{array}$} \\
\hline & \multirow{3}{*}{$\begin{array}{l}\text { Anaesthesiologists } \\
\text { Certified registered nurse anaesthetists } \\
\text { Surgeons }\end{array}$} & Reinforcement & \\
\hline & & $\begin{array}{l}\text { Environmental } \\
\text { context and resources }\end{array}$ & \\
\hline & & $\begin{array}{l}\text { Social/professional } \\
\text { role and identity }\end{array}$ & $\begin{array}{l}\text { Role delegation: } \\
\text { - Anaesthesiologist or certified registered nurse anaesthetist } \\
\text { responsible for validating the POCEP }\end{array}$ \\
\hline \multirow{2}{*}{$\begin{array}{l}\text { Shapiro et al. } \\
\text { (2018) [68] } \\
\text { USA }\end{array}$} & Quasi-experimental (pre-post) & $\begin{array}{l}\text { Environmental } \\
\text { context and resources }\end{array}$ & \multirow{2}{*}{$\begin{array}{l}\text { Educational services: } \\
\text { - Educational sessions highlighting current recommendations: } \\
\text { presentation of current practice highlighting antibiotic usage } \\
\text { (transparency of practice) }\end{array}$} \\
\hline & Gynaecological surgeons & Knowledge & \\
\hline \multirow{2}{*}{$\begin{array}{l}\text { Sutherland et al. } \\
\text { (2014) [69] } \\
\text { USA }\end{array}$} & Quasi-experimental (pre-post) & $\begin{array}{l}\text { Behavioural } \\
\text { regulation }\end{array}$ & \multirow{2}{*}{$\begin{array}{l}\text { Audit and feedback and multidisciplinary collaboration: } \\
\text { - Multidisciplinary involvement in feedback committee } \\
\text { - Discussions/contact made with staff who are constantly non- } \\
\text { compliant to review practice }\end{array}$} \\
\hline & $\begin{array}{l}\text { Anaesthesiologists } \\
\text { Surgeons }\end{array}$ & Social influences & \\
\hline \multirow{4}{*}{$\begin{array}{l}\text { Telfah et al. } \\
\text { (2015) [70] } \\
\text { Jordan }\end{array}$} & Quasi-experimental (pre-post) & Knowledge & \multirow{2}{*}{$\begin{array}{l}\text { Educational services: } \\
\text {-Revising guidelines to ensure it is comprehensive then providing } \\
\text { education to clinical staff to increase awareness } \\
\text {-One to one educational sessions and email reminders also } \\
\text { provided }\end{array}$} \\
\hline & $\begin{array}{l}\text { Medical, nursing and pharmacy staff } \\
\text { Surgical residents }\end{array}$ & $\begin{array}{l}\text { Environmental } \\
\text { context and resources }\end{array}$ & \\
\hline & & $\begin{array}{l}\text { Social/professional } \\
\text { role and identity }\end{array}$ & $\begin{array}{l}\text { Role delegation: } \\
\text { - Assigning a clinical pharmacist to review and evaluate } \\
\text { prescribed medications to ensure compliance }\end{array}$ \\
\hline & & $\begin{array}{l}\text { Environmental } \\
\text { context and resources }\end{array}$ & $\begin{array}{l}\text { Other enablers: } \\
\text {-Creation of a theatre satellite pharmacy to allow pharmacists to } \\
\text { review and process all orders prior to supply, whilst also } \\
\text { restricting access to antibiotics }\end{array}$ \\
\hline
\end{tabular}


Table 2 Summary of reported enablers (Continued)

\begin{tabular}{|c|c|c|c|}
\hline $\begin{array}{l}\text { Author } \\
\text { (year) and } \\
\text { country }\end{array}$ & Study design and population & TDF Domain & Description of reported enablers \\
\hline \multirow{3}{*}{$\begin{array}{l}\text { Wax et al. } \\
\text { (2007) [71] } \\
\text { USA }\end{array}$} & Quasi-experimental (pre-post) & Reinforcement & \multirow{3}{*}{$\begin{array}{l}\text { Real-time reminders: } \\
\text {-Activation of a visual reminder on the AIMS to ensure antibiotics } \\
\text { are given before incision }\end{array}$} \\
\hline & \multirow{2}{*}{$\begin{array}{l}\text { Anaesthesia care team (certified registered nurse } \\
\text { anaesthetist, anaesthesia house staff and } \\
\text { attending anaesthesiologist) }\end{array}$} & $\begin{array}{l}\text { Behavioural } \\
\text { regulation }\end{array}$ & \\
\hline & & $\begin{array}{l}\text { Environmental } \\
\text { context and resources }\end{array}$ & \\
\hline \multirow[t]{3}{*}{$\begin{array}{l}\text { Whitman et al. } \\
\text { (2008) [72] } \\
\text { USA }\end{array}$} & Quasi-experimental (pre-post) & $\begin{array}{l}\text { Social/professional } \\
\text { role and identity }\end{array}$ & $\begin{array}{l}\text { Role delegation: } \\
\text { - Anaesthetists assumed responsibility of antibiotic administration } \\
\text { in theatre }\end{array}$ \\
\hline & \multirow[t]{2}{*}{ Anaesthetists } & $\begin{array}{l}\text { Behavioural } \\
\text { regulation }\end{array}$ & \multirow{2}{*}{$\begin{array}{l}\text { Support tools: } \\
\text { - Use of a preoperative order form in preadmission clinic to } \\
\text { ensure antibiotics are charted early } \\
\text { Other enablers: } \\
\text { - Ensuring patients don't leave hold area until antibiotics are } \\
\text { administered }\end{array}$} \\
\hline & & $\begin{array}{l}\text { Environmental } \\
\text { context and resources }\end{array}$ & \\
\hline \multirow{2}{*}{$\begin{array}{l}\text { Willems et al. } \\
\text { (2005) [73] } \\
\text { Belgium }\end{array}$} & Quasi-experimental (pre-post) & \multirow{2}{*}{$\begin{array}{l}\text { Behavioural } \\
\text { regulation }\end{array}$} & \multirow{2}{*}{$\begin{array}{l}\text { Audit and feedback: } \\
\text { - Highlighting cost to hospital when SAP not adhered to (use of } \\
\text { a follow up form to highlight cost of antibiotics when following } \\
\text { SAP guidelines vs the antibiotic regimen used by the doctor) }\end{array}$} \\
\hline & Physicians & & \\
\hline \multirow{3}{*}{$\begin{array}{l}\text { Zanetti et al. } \\
\text { (2003) [74] } \\
\text { USA }\end{array}$} & Randomised control trial & $\begin{array}{l}\text { Environmental } \\
\text { context and resources }\end{array}$ & \multirow{3}{*}{$\begin{array}{l}\text { Real-time reminders: } \\
\text { - Use of an audible alarm (computer generated) to notify staff } \\
\text { when antibiotic needs to be re-dosed as well as pop-up notifi- } \\
\text { cation displaying re-dosing guidelines }\end{array}$} \\
\hline & \multirow[t]{2}{*}{ Surgical staff } & $\begin{array}{l}\text { Behavioural } \\
\text { regulation }\end{array}$ & \\
\hline & & Reinforcement & \\
\hline \multirow{2}{*}{$\begin{array}{l}\text { Zanotto et al. } \\
\text { (2006) [75] } \\
\text { Brazil }\end{array}$} & Quasi-experimental (pre-post) & $\begin{array}{l}\text { Behavioural } \\
\text { regulation }\end{array}$ & \multirow{2}{*}{$\begin{array}{l}\text { Support tools: } \\
\text { - Placing software restrictions on certain antibiotics to limit } \\
\text { inappropriate prescribing } \\
\text { - Interruption of dispensing if no reason documented } \\
\text { electronically to justify extended antibiotic use }\end{array}$} \\
\hline & Not specified & $\begin{array}{l}\text { Environmental } \\
\text { context and resources }\end{array}$ & \\
\hline \multirow[t]{4}{*}{$\begin{array}{l}\text { Zhou et al. } \\
\text { (2016) [76] } \\
\text { China }\end{array}$} & Quasi-experimental (pre-post) & $\begin{array}{l}\text { Social/professional } \\
\text { role and identity }\end{array}$ & $\begin{array}{l}\text { Role delegation: } \\
\text { - Delegating task of reviewing antimicrobial prescribing to clinical } \\
\text { pharmacist }\end{array}$ \\
\hline & \multirow{3}{*}{$\begin{array}{l}\text { Pharmacists } \\
\text { Surgeons } \\
\text { Nurses }\end{array}$} & Knowledge & \multirow{2}{*}{$\begin{array}{l}\text { Educational services: } \\
\text { - Educational sessions to medical, surgical and nursing staff } \\
\text { regarding appropriate SAP }\end{array}$} \\
\hline & & $\begin{array}{l}\text { Environmental } \\
\text { context }\end{array}$ & \\
\hline & & $\begin{array}{l}\text { Behavioural } \\
\text { regulation }\end{array}$ & $\begin{array}{l}\text { Audit and feedback: } \\
\text { - Weekly performance reports regarding SAP adherence and } \\
\text { irrational antibiotic use } \\
\text { - Communication between staff when inappropriate prescribing } \\
\text { detected }\end{array}$ \\
\hline
\end{tabular}

Abbreviations: AIMS Anaesthesia Information Management System, CPOE computerised physician order entry, EMR electronic medical record, POCEP point-of-care electronic prompt, SAM Smart Anaesthesia Manager, SAP surgical antimicrobial prophylaxis, SSI surgical site infection

highlighting the need for active methods of education to be utilised in a hospital setting $[87,88]$.

Guidelines contain the most current evidence based research [15] and can often act as a means of education; however, inaccessibility can act as a hindrance to its use. Guidelines that were inaccessible, in print or electronic form, presented a concern to hospital staff. In some instances, guidelines were completely inaccessible in theatre or on the wards, preventing its use by clinical staff such as surgeons, ward nurses and anaesthesia personnel (anaesthetists and nurse anaesthetists), while in other instances it was hard to locate [36, 79]. Whilst guideline dissemination has been reported as an enabler to SAP guideline adherence, it is important to note that the method of guideline dissemination and presentation also plays a role in determining the likely uptake of content [28]. Guidelines should be presented in a simple, user friendly format that reduces the time required to search for information [28]. An example of a successful intervention present in the review is the use of a "traffic light" poster system which assists staff in determining antibiotic choice, dose and duration of prophylaxis for various gastrointestinal surgical procedures [43]. By providing a colour coded visual reminder in theatre, staff were able to easily refer to guideline information when required, thus resulting in a 2 fold improvement in guideline adherence [43]. 
Following on, the location of guidelines needs to be considered. In order to make use of SAP guidelines, they need to be present in a location that is readily accessible [28]. The presence of guidelines in operating theatres assists in the uptake of guidelines due to the ease in which it can be accessed [42, 43, 45, 47].

Organisational barriers and the local context can play a role in preventing appropriate adherence to SAP guidelines. The local setting, particularly the culture and practice of staff can influence how SAP guidelines are used. Often, behaviour, beliefs and assumptions are shared by staff in an organisation; however when culture affects performance negatively, staff may become "entrapped” leading to poorer practice $[89,90]$. This highlights how settings where improvisation is the norm can heavily impact the extent of guideline incorporation into clinical practice [37]. Furthermore, a lack of agreement of guideline content by health care professionals can result in limited use of guidelines [78], exemplifying the value of including stakeholders in guideline development [78]. Involving key stakeholders in guideline development can increase the likelihood of guidelines being adhered to $[79,85]$, thus resulting in less variation in how practice is carried out.

Health care professionals often work in settings that are fast-paced, in which there is a high workload and limited resources [91]. Excessive workloads and time constraints place undue pressure on staff, resulting in changes to workflow and preventing staff from providing the best possible care to their patients $[36,92]$. One such example is the effect of workflow on the timing of SAP administration. In a qualitative study by Tan et al. [40], participants noted that the unpredictability of workflow systems negatively impacts the timing of antibiotic administration, with the potential to compromise quality standards within hospitals. This is further noted in the study by Al-Azzam et al. [35], in which one third (33\%) of participants stated that work flow was a contributing factor to inappropriate timing of SAP administration.

In situations where time is short, health care professionals are more likely to resort to intuitive processes or past practice and experience as a guiding point for their practice rather than rely on and implement recommendations in guidelines [91]. Old habits as well as previous training often overrides the use of evidence-based medicine in these situations, highlighting the need to ensure appropriate time and work allocation to staff [28].

\section{Skills and beliefs about capabilities}

Another barrier to SAP guideline adherence was the reliance on habits picked up during training to guide practice [39]. Overall, 25\% of participants in Madubueze et al.'s study [39] indicated that old practices taught in training was a reason for not adhering to SAP guidelines, with nearly half (44\%) of participants in the youngest age group surveyed indicating this was also the cause. It was also noted by Broom et al. [37] that junior doctors were more likely to request inappropriate SAP (such as using antibiotics in procedures where it is not required) than senior doctors. Interestingly, the opposite has also been noted. In a mixed methods study by Giusti et al. [78], participants with more than 18 years of practice stated that SAP was determined by personal experience rather than guideline content. In a qualitative study by Ierano et al. [85], it was perceived that younger surgeons were more likely to access guidelines; however, final decisions on antibiotic use was ultimately decided by senior staff. Participants in this study mentioned they did not feel empowered to speak up or challenge senior consultants as it was believed that this would impact their future careers [85].

Effective communication is an important skill required by all health professionals, particularly when practising in a multidisciplinary environment [93]. Effective communication can allow for improved patient safety, greater employee morale and greater flow of information [93]. Thus, enhancing communication between staff such as through comprehensive preoperative team briefings [57] and ensuring consultations take place between staff prior to decision making [41] can help improve guideline adherence.

Social/professional role and identity and Social influences Interpersonal barriers were present between staff from multiple specialties, with many presenting between the surgeon and anaesthetist. Lack of communication can lead to SAP mismanagement particularly at induction of anaesthesia, if roles of staff are not clearly determined [35, 36, 40, 77]. Without delegating the task of SAP prescribing and administration, patients may fail to receive the required antibiotic at the relevant time [40]. However, in situations where the role is clear, medical hierarchy can often place a strain or tension on the relationship [77]. Challenging decisions made by senior staff within the same specialty or between specialties is difficult, with many health professionals feeling uncomfortable in doing so [85]. This is especially the case with junior staff, who feel more inclined to accept senior recommendations despite it not aligning with guidelines in an effort to preserve relationships and their career [77, 85].

Role delegation was noted to be an enabler in many studies $[41,49,60,62,65,67,70,72,76]$. Whilst surgeons often took responsibility for prescribing SAP [49, $85]$, the role of antibiotic administration was delegated to either the anaesthetist or nurse $[41,49,60,62,65,67$, 72]. The correct timing of preoperative administration is 
Table 3 Summary of studies that discussed both barriers and enablers

\begin{tabular}{|c|c|c|c|c|c|}
\hline $\begin{array}{l}\text { Author } \\
\text { (year) } \\
\text { and } \\
\text { country }\end{array}$ & $\begin{array}{l}\text { Study design } \\
\text { and population }\end{array}$ & TDF Domain & Description of reported barrier & TDF Domain & Description of reported enabler \\
\hline \multirow[t]{8}{*}{$\begin{array}{l}\text { Broom } \\
\text { et al. } \\
\text { (2018) } \\
\text { [77] } \\
\text { Australia }\end{array}$} & Qualitative & $\begin{array}{l}\text { Social/ } \\
\text { professional } \\
\text { role and } \\
\text { identity }\end{array}$ & \multirow{2}{*}{$\begin{array}{l}\text { Personal barriers (interpersonal): } \\
\text { Relationship between surgeon and } \\
\text { anaesthetist: } \\
\text { - Poor communication between surgeons } \\
\text { and anaesthetist } \\
\text { - Lack of task delegation in regards to } \\
\text { antibiotic use }\end{array}$} & Skills & \multirow[t]{8}{*}{$\begin{array}{l}\text { Effective communication: } \\
\text { - Working in a private hospital sector as } \\
\text { communication was seen as better. } \\
\text { Improved responsibility sharing } \\
\text { between surgeon and anaesthetist }\end{array}$} \\
\hline & \multirow[t]{7}{*}{$\begin{array}{l}\text { Surgeons } \\
\text { Anaesthetists }\end{array}$} & $\begin{array}{l}\text { Social } \\
\text { influences }\end{array}$ & & \multirow[t]{7}{*}{$\begin{array}{l}\text { Environmental } \\
\text { context and resources }\end{array}$} & \\
\hline & & $\begin{array}{l}\text { Environmental } \\
\text { context and } \\
\text { resources }\end{array}$ & $\begin{array}{l}\text { Hierarchy within and between surgical } \\
\text { and anaesthetist teams: } \\
\text { - Hierarchy affects whether a colleague's } \\
\text { decision would be "challenged" }\end{array}$ & & \\
\hline & & $\begin{array}{l}\text { Beliefs about } \\
\text { capabilities }\end{array}$ & \multirow{3}{*}{$\begin{array}{l}\text { Personal barrier (intrapersonal): } \\
\text { - Surgeon level of experience influences } \\
\text { whether or not they choose to prescribe } \\
\text { SAP (junior vs senior staff) }\end{array}$} & & \\
\hline & & Skills & & & \\
\hline & & Knowledge & & & \\
\hline & & $\begin{array}{l}\text { Environmental } \\
\text { context and } \\
\text { resources }\end{array}$ & \multirow{2}{*}{$\begin{array}{l}\text { Organisational barriers: } \\
\text { - Workflow - especially emergency } \\
\text { settings, communication and } \\
\text { consultation may not occur. SAP may } \\
\text { not be considered a priority } \\
\text { - Effect of influential staff members on } \\
\text { local cultures of prescribing (again the } \\
\text { effect of hierarchy influencing correct } \\
\text { SAP use) }\end{array}$} & & \\
\hline & & $\begin{array}{l}\text { Social } \\
\text { influences }\end{array}$ & & & \\
\hline \multirow[t]{5}{*}{$\begin{array}{l}\text { Giusti } \\
\text { et al. } \\
\text { (2016) [78] } \\
\text { Italy }\end{array}$} & Mixed methods & $\begin{array}{l}\text { Environmental } \\
\text { context and } \\
\text { resources }\end{array}$ & \multirow{4}{*}{$\begin{array}{l}\text { Personal barriers (intrapersonal): } \\
\text { - Disagreement between health care } \\
\text { professionals and content in guidelines } \\
\text { - Belief that antibiotics listed in guidelines } \\
\text { are not efficacious } \\
\text { - Individual understanding of the meaning } \\
\text { of prophylaxis; poorer understanding } \\
\text { meant that antibiotic use was extended } \\
\text { as a precautionary measure } \\
\text { - Poor knowledge of local hospital data } \\
\text { on how SAP is used and the incidence } \\
\text { of SSIs } \\
\text { - Level of experience: older, more } \\
\text { experienced staff more likely to follow } \\
\text { personal experience over guidelines }\end{array}$} & Knowledge & $\begin{array}{l}\text { Guideline dissemination: } \\
\text { - Dissemination of guidelines, particularly } \\
\text { when shared and communicated }\end{array}$ \\
\hline & \multirow{4}{*}{$\begin{array}{l}\text { Anaesthesiologists } \\
\text { Surgeons } \\
\text { Nurse } \\
\text { coordinators }\end{array}$} & Knowledge & & $\begin{array}{l}\text { Environmental } \\
\text { context and resources }\end{array}$ & \\
\hline & & $\begin{array}{l}\text { Beliefs about } \\
\text { capabilities }\end{array}$ & & Social influences & $\begin{array}{l}\text { Multidisciplinary collaboration: } \\
\text { - Trust in guideline developers. } \\
\text { Multidisciplinary collaboration to } \\
\text { develop guidelines }\end{array}$ \\
\hline & & & & $\begin{array}{l}\text { Beliefs about } \\
\text { consequences }\end{array}$ & $\begin{array}{l}\text { Other enablers: } \\
\text { - Belief that guideline adherence can act } \\
\text { as a protective tool if legal action is } \\
\text { taken against practitioner }\end{array}$ \\
\hline & & $\begin{array}{l}\text { Environmental } \\
\text { context and } \\
\text { resources }\end{array}$ & $\begin{array}{l}\text { External barriers: } \\
\text { - Parental expectation that SAP would } \\
\text { be used } \\
\text { - Pharmaceutical company pressure in } \\
\text { regards to choice of antibiotic } \\
\text { Organisational barriers: } \\
\text { - Availability of hand hygiene facilities - } \\
\text { overcrowding of patient rooms during } \\
\text { visiting hours can lead to extended } \\
\text { prophylaxis }\end{array}$ & & \\
\hline \multirow[t]{3}{*}{$\begin{array}{l}\text { Nobile } \\
\text { et al. } \\
\text { (2014) [79] } \\
\text { Italy }\end{array}$} & $\begin{array}{l}\text { Quasi- } \\
\text { experimental } \\
\text { (pre-post), } \\
\text { Quantitative } \\
\text { descriptive }\end{array}$ & $\begin{array}{l}\text { Environmental } \\
\text { context and } \\
\text { resources }\end{array}$ & \multirow[t]{3}{*}{$\begin{array}{l}\text { Organisational barrier: } \\
\text { - Lack of guideline presence on wards }\end{array}$} & $\begin{array}{l}\text { Social } \\
\text { influences }\end{array}$ & $\begin{array}{l}\text { Multidisciplinary collaboration: } \\
\text { - Collaboration to review and update } \\
\text { existing guidelines }\end{array}$ \\
\hline & $\begin{array}{l}\text { Orthopaedic } \\
\text { surgeons }\end{array}$ & & & Knowledge & $\begin{array}{l}\text { Educational services: } \\
\text { - Educational sessions to explain SSI } \\
\text { prevention, }\end{array}$ \\
\hline & $\begin{array}{l}\text { Nurses } \\
\text { Pharmacists }\end{array}$ & & & $\begin{array}{l}\text { Environmental } \\
\text { context and } \\
\text { resources }\end{array}$ & $\begin{array}{l}\text { guidelines as well as the correct } \\
\text { administration of SAP } \\
\text { Guideline dissemination: } \\
\text { - Development of pocket sized } \\
\text { guidelines for quick reference }\end{array}$ \\
\hline
\end{tabular}


Table 3 Summary of studies that discussed both barriers and enablers (Continued)

\begin{tabular}{|c|c|c|c|c|c|}
\hline $\begin{array}{l}\text { Author } \\
\text { (year) } \\
\text { and } \\
\text { country }\end{array}$ & $\begin{array}{l}\text { Study design } \\
\text { and population }\end{array}$ & TDF Domain & Description of reported barrier & TDF Domain & Description of reported enabler \\
\hline & & & & Behavioural regulation & $\begin{array}{l}\text { Audit and feedback: } \\
\text { - Feedback given to staff when deviation } \\
\text { from practice detected (regular } \\
\text { monitoring and evaluation of practice) }\end{array}$ \\
\hline
\end{tabular}

important as evidence suggests that administration of antibiotics greater than 120 min prior to incision is associated with a significantly higher risk of SSIs [8]. Thus, it is crucial to ensure that serum and tissue concentrations of antibiotics are adequate at time of incision [14]. As anaesthetists are responsible for administering anaesthesia in theatre as well as other medications [94], many studies found that delegating the role of antibiotic administration to anaesthetists assisted with timely provision of the preoperative dose [41, 60, 62, 72]. In an effort to improve adherence to SAP guidelines, Whitman et al. [72] employed a number of interventions that resulted in an increase in adherence from 55 to $90 \%$. After delegating the role of antibiotic administration to the anaesthetist, this adherence increased further to $95 \%$.
Pharmacists were generally assigned the role of reviewing the appropriateness of prescribed antibiotics to ensure that drug choice and duration of use was suitable [70, 76]. Zhou et al. [76] found that by delegating a pharmacist to review SAP prescribing, adherence to guidelines increased from 83 to $92.2 \%$.

\section{Emotion and beliefs about consequences}

Fear of repercussion and concerns of medical malpractice can drive the overuse of antibiotics, thus resulting in a deviation from standard practice. Although guidelines advocate limiting antibiotic use for surgical prophylaxis where appropriate, the undesirable consequences that can arise from SSI development such as increased morbidity and mortality, hospital readmissions and increased

Table 4 Interventions employed that did not influence guideline adherence

\begin{tabular}{|c|c|c|c|}
\hline $\begin{array}{l}\text { Author } \\
\text { (year) } \\
\text { and } \\
\text { country }\end{array}$ & $\begin{array}{l}\text { Study design and } \\
\text { population }\end{array}$ & Intervention & Potential reasons for outcome \\
\hline $\begin{array}{l}\text { Knox and } \\
\text { Edye } \\
\text { (2016) [80] } \\
\text { Australia }\end{array}$ & $\begin{array}{l}\text { Quasi-experimental } \\
\text { (pre-post) } \\
\text { Not specified }\end{array}$ & $\begin{array}{l}\text { Education and increasing awareness without } \\
\text { attempting to change practice } \\
\text { - Display of SAP guidelines for majority of the } \\
\text { surgical procedures in surgical areas-mainly in } \\
\text { theatre. Information present included the } \\
\text { recommended drug, dose, time and duration } \\
\text { - Substantial advertising throughout the hospital } \\
\text { site to raise general awareness of appropriate } \\
\text { prescribing of antibiotics in all clinical areas }\end{array}$ & $\begin{array}{l}\text { Knox and Edye [80] believe low uptake may be due to } \\
\text { cognitive dissonance as the educational interventions } \\
\text { used were passive in nature }\end{array}$ \\
\hline
\end{tabular}

Nemeth Quasi-experimental et al. (pre-post)

(2010) [81] Anaesthesia, nursing

USA $\quad$ anaesthesia, nursing

- Education of anaesthesia, surgical and nursing staff for a one month period

- Modification of pre-operative checklist to include confirmation of timely antibiotic administration
Nemeth et al. [81] believe that results were lower in the post-intervention group due to:

(a) Pre-operative verification not being conducted

(b) Verification being conducted incorrectly

(c) An inappropriate response or lack of response to verification

Furthermore, pre-intervention compliance rates were quite high (90\%) and sustained effects of intervention could not be observed due to short duration of postintervention period (5 days)

Putnam Quasi-experimental et al. (pre-post)

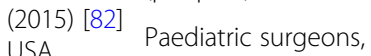

USA anaesthesiologists and peri-operative staff

(a) Little effort in disseminating the CPOE

\footnotetext{
- Pre-operative checklist modification to ensure antibiotics are correctly administered

- CPOE used so that physicians can order antibiotics from pharmacy at any point prior to procedure

- Role delegation - anaesthetists responsible for administering antibiotics

- Attachment of guidelines to anaesthesia carts in theatre

- Revised guidelines disseminated electronically to all peri-operative staff
}

Putnam et al. [82] believe that outcomes were poor due to:

(b) Minimal education being provided on how to use the program

(c) Lack of monitoring of CPOE use after implementation

(d) Poor dissemination and implementation of the intervention cycles and guidelines 
economic burden can result in an extended duration of prophylaxis [37, 78, 85, 95]. Providing additional doses of antibiotics usually adds a perceived layer of comfort for surgeons, thus acting as a line of defence for the surgeon in the situation where a patient develops an infection [37].

\section{Memory, attention and decision processes, behavioural regulation and reinforcement}

To ensure adequate serum and tissue concentrations, intraoperative re-dosing is recommended in SAP guidelines when the duration of a procedure exceeds two half-lives of the administered preoperative drug or if there is excessive blood loss [14]. However, the administration of SAP has been considered low priority during complex and lengthy procedures [37, 40, 77, 85]. Due to the complex procedure at hand, staff may "forget" the need to readminister antibiotics, thus preventing a patient from receiving adequate prophylaxis due to the under-administration of antibiotics [37, 40]. Memory and attention levels can impact the extent to which guidelines are adhered to. Therefore, the use of real-time information, such as electronic prompts and reminders can reinforce guideline information and dramatically increase the level of adherence with SAP guidelines. Nair et al. [58] found that using a real-time feedback and reminder system increased the compliance of timely administration of pre-incisional antibiotics to nearly $100 \%$. In a second study by Nair et al. [59] to determine the impact of electronic reminders on intraoperative redosing of antibiotics, the authors found that a real-time reminder system improved the rate of intraoperative redosing from 62.5 to $\sim 84 \%$.

The practice of audit and feedback is a popular strategy often implemented in health care settings in order to modify behaviour. As evident by the studies included in this review $[41,45,47,48,50,55,56,58,60,64,69,73,76$, 79], the coupling of audits with a feedback mechanism generally results in an increased adherence to SAP guidelines. In the study by Sutherland et al. [69], direct physician to physician feedback was used in order to improve adherence to SAP guidelines. Sutherland et al. [69] found that by involving both surgeons and anaesthetists in feedback committees, repeat errors regarding selection and administration of antibiotics could be reduced, thus resulting in an increased adherence to SAP guidelines. It is crucial, however, to ensure that surveillance occurs at regular intervals in order to enhance behaviour change. This provides an opportunity to uncover reasons behind poor guideline adherence whilst allowing for the development of behavioural change strategies. Providing feedback to staff in relation to their practice allows for reflection to take place, thereby resulting in corrective action or motivation to continue performing at the same or a higher level [60]. On a larger scale, presenting results or benchmarking between hospital units or regional districts can lead to competition which can enhance uptake of guidelines at a local setting $[41,85]$.

Although audit and feedback is seen as a successful means of changing practice, it is important to note this is dependent on factors such as baseline performance of staff and the methods in which feedback is provided [96]. A Cochrane systematic review published in 2012 [96] highlights that, if designed well and used in the right context, audit and feedback can help improve practice. Although the outcomes of this review suggests that the effect of audit and feedback is small to moderate, the effect of feedback can be higher if baseline performance is low, feedback is provided by a supervisor on a regular basis, if feedback is issued in both verbal and written form and an action plan is provided to staff [96].

Papers that reported successful interventions often used a bundle of interventions to change practice. Although the literature contains abundant examples on the benefits of multifaceted interventions in enhancing guideline use [28, 88, 97, 98], Grimshaw and Eccles [99] argue that this may not always be the case. This finding is drawn from a comprehensive systematic review conducted by Grimshaw et al. [100] in which robust statistical techniques were used to make comparisons between intervention types. Grimshaw et al. [100] highlight that previous reviews comparing interventions often used vote-counting to determine effect, whilst providing minimal information regarding effect sizes of interventions. Vote-counting has been used to determine the effectiveness of interventions by comparing the number of positive outcomes to the number of negative outcomes; however, issues may arise by using this technique as it does not provide information on the magnitude of effects [100, 101].

A previous review was conducted by $\mathrm{Ng}$ and Chong in 2012 [102] to identify factors that influence a surgeon's adherence to SAP guidelines. Of note, a lack of awareness and ineffective dissemination of guidelines (such as updating guidelines in a hospital's handbook without removing old guidelines from theatre) was considered a pivotal reason behind poor guideline use [102, 103]. The review also noted that the use of education and audit and feedback often enhanced guideline adherence [102]. While Ng and Chong's review [102] specifically highlighted the factors that affect a surgeon's adherence to guidelines, it did not consider the role of other health professionals in the optimal use of SAP guidelines. In addition, a review by Gouvea et al. in 2015 [104] analysed the adherence rates to SAP guidelines, however, did not undertake an exploration of the factors that resulted in poor adherence.

The strengths of this review include the systematic search of multiple databases through a rigorous search strategy and 
the evaluation of quality of included studies. The vast majority of papers included were deemed to be of high quality. Through this review, we were able to shed light on the TDF domains that have arisen through the various studies mentioned here, highlighting the factors that affect guideline adherence rates. We focused on identifying the reported barriers and enablers to SAP guideline adherence rather than factors that were perceived or speculated by authors throughout the discussion of their studies. Given the multidisciplinary involvement in SAP management, we did not exclude any health professionals from our review. Through this, we were able to highlight how the practice of surgeons, anaesthetists, nurses and pharmacists contributes to the uptake of guidelines.

Of the limitations of this review, we excluded articles that were in a language other than English which may have limited our results to some extent. We also did not examine the grey literature such as theses or conference abstracts which may discuss the outcomes of quality improvement projects on the effect of SAP guideline adherence. However, we were still able to retrieve a large number of relevant studies from our search. Furthermore, we did not consider studies where guidelines were introduced as part of the intervention. Thus, we were unable to ascertain whether SAP guideline adherence was greater when newly developed guidelines were coupled with other interventions.

\section{Conclusions}

Multiple factors contribute to the suboptimal adherence to SAP guidelines. It is clear that there is a need to identify the factors that may prevent the uptake of guidelines in a local setting, whilst also determining interventions that not only enhance the adherence rates but sustain it for an extended period of time in order to modify practice. Successful studies often employ the use of multiple interventions simultaneously, highlighting the importance of combining different means to change practice. The importance of avoiding passive methods to disseminate information is also clear as the engagement of key stakeholders is crucial to developing change. By understanding the local environment and the nuances that pertain to it, theoretically derived interventions can be developed and implemented, thus increasing the likelihood of adhering to SAP guidelines.

\section{Supplementary Information}

The online version contains supplementary material available at https://doi. org/10.1186/s13643-021-01577-w.

Additional file 1. PRISMA 2009 Checklist.

Additional file 2. Search strategy.

Additional file 3. List of studies excluded from systematic review.

Additional file 4. Quality assessment using the Mixed Methods Appraisal Tool (MMAT) Version 2018.

\section{Abbreviations}

AIMS: Anaesthesia Information Management System; CDC: Centers for Disease Control and Prevention; CPOE: Computerised physician order entry EMR: Electronic medical record; MMAT: Mixed method appraisal tool; PRIS MA: Preferred Reporting Items for Systematic Reviews and Meta-Analyses; POCEP: Point of care electronic prompt; SAM: Smart Anaesthesia Manager; SAP: Surgical antimicrobial prophylaxis; SSIs: Surgical site infections;

WHO: World Health Organization

\section{Acknowledgements}

The authors would like to acknowledge Karen Macvean for her assistance in developing the search strategy.

\section{Authors' contributions}

SH, VC, JS, IS conceived the review. SH developed the search strategy and undertook the initial screening of all titles and abstracts. Full text articles were reviewed by SH and IS. Where consensus could not be reached, articles were reviewed by VC and JS. All authors participated in the initial quality assessment of included studies. SH drafted the manuscript which was reviewed and revised by VC, JS and IS. All authors read and approved of the final manuscript.

\section{Funding}

The authors have no funding sources to declare.

\section{Availability of data and materials}

All data generated or analysed during this study are included in this published article [and its supplementary information files].

Ethics approval and consent to participate

Not applicable.

Consent for publication

Not applicable.

\section{Competing interests}

The authors declare that they have no competing interests.

\section{Author details}

${ }^{1}$ Pharmacy, School of Health and Biomedical Sciences, RMIT University, Bundoora, Victoria, Australia. ${ }^{2}$ School of Pharmacy and Medical Sciences, University of South Australia, Adelaide, South Australia, Australia. ${ }^{3}$ Adelaide Medical School, Faculty of Health and Medical Sciences, University of Adelaide, Adelaide, South Australia, Australia.

Received: 19 July 2020 Accepted: 2 January 2021

Published online: 16 January 2021

\section{References}

1. Uckay I, Harbarth S, Peter R, Lew D, Hoffmeyer P, Pittet D. Preventing surgical site infections. Expert Rev Anti Infect Ther. 2010;8(6):657-70. https:// doi.org/10.1586/eri.10.41 Epub 2010/06/05. PubMed PMID: 20521894.

2. Centers for Disease Control and Prevention. In: CDC, editor. Surgical Site Infection (SSI) event. Atlanta; 2020. [updated January 2020; cited 2020 April 6]. Available from: https://www.cdc.gov/nhsn/pdfs/pscmanual/ 9pscssicurrent.pdf.

3. Mangram AJ, Horan TC, Pearson ML, Silver LC, Jarvis WR. Guideline for prevention of surgical site infection, 1999. Am J Infect Control. 1999;27(2): 97-134. https://doi.org/10.1016/S0196-6553(99)70088-X.

4. Zimlichman E, Henderson D, Tamir O, Franz C, Song P, Yamin CK, et al. Health care-associated infections: a meta-analysis of costs and financial impact on the US health care system. JAMA Intern Med. 2013;173(22):203946. https://doi.org/10.1001/jamainternmed.2013.9763.

5. Broex ECJ, van Asselt ADI, Bruggeman CA, van Tiel FH. Surgical site infections: how high are the costs? J Hosp Infect. 2009:72(3):193-201. https://doi.org/10.1016/j.jhin.2009.03.020.

6. Badia JM, Casey AL, Petrosillo N, Hudson PM, Mitchell SA, Crosby C. Impact of surgical site infection on healthcare costs and patient outcomes: a systematic review in six European countries. J Hosp Infect. 2017:96(1):1-15. https://doi.org/10.1016/j.jhin.2017.03.004. 
7. lerano C, Manski-Nankervis J, James R, Rajkhowa A, Peel T, Thursky K. Surgical antimicrobial prophylaxis. Aust Prescr. 2017;40:225-9. https://doi. org/10.18773/austprescr.2017.073.

8. Allegranzi B, Bischoff $\mathrm{P}$, de Jonge S, Kubilay NZ, Zayed B, Gomes SM, et al. New WHO recommendations on preoperative measures for surgical site infection prevention: an evidence-based global perspective. Lancet Infect Dis. 2016;16(12):e276-e87. https://doi.org/10.1016/S1473-3099(16)30398-X.

9. Allegranzi B, Zayed B, Bischoff P, Kubilay NZ, de Jonge S, de Vries F, et al. New $\mathrm{WHO}$ recommendations on intraoperative and postoperative measures for surgical site infection prevention: an evidence-based global perspective. Lancet Infect Dis. 2016;16(12):e288-303. https://doi.org/10.1016/S14733099(16)30402-9.

10. Gillespie WJ, Walenkamp G. Antibiotic prophylaxis for surgery for proximal femoral and other closed long bone fractures. Cochrane Database Syst Rev. 2001;(1):Cd000244. https://doi.org/10.1002/14651858.Cd000244 Epub 2001/ 05/02. PubMed PMID: 11279687.

11. Bryson DJ, Morris DL, Shivji FS, Rollins KR, Snape S, Ollivere BJ. Antibiotic prophylaxis in orthopaedic surgery: difficult decisions in an era of evolving antibiotic resistance. Bone Joint J. 2016:98-b(8):1014-9. https://doi.org/10. 1302/0301-620x.98b8.37359 Epub 2016/08/03. PubMed PMID: 27482011.

12. Berrios-Torres SI, Umscheid CA, Bratzler DW, Leas B, Stone EC, Kelz RR, et al. Centers for disease control and prevention guideline for the prevention of surgical site infection, 2017. JAMA Surg. 2017;152(8):784-91. https://doi.org/ 10.1001/jamasurg.2017.0904 Epub 2017/05/04. PubMed PMID: 28467526.

13. Australian Commission on Safety and Quality in Health Care. Antimicrobial stewardship clinical care standard. Sydney: ACSQHC; 2014.

14. Bratzler DW, Dellinger EP, Olsen KM, Perl TM, Auwaerter PG, Bolon MK, et al. Clinical practice guidelines for antimicrobial prophylaxis in surgery. Am J Health Syst Pharm. 2013;70(3):195-283. https://doi.org/10.2146/ajhp120568 Epub 2013/01/19. PubMed PMID: 23327981.

15. Institute of Medicine (US) Committee to Advise the Public Health Service on Clinical Practice Guidelines. Definitions of key terms. In: Clinical practice guidelines: directions for a new program. Washington (DC): National Academies Press (US); 1990. Available from: https://www.ncbi.n/m.nih.gov/ books/NBK235758/.

16. Musmar SM, Ba'ba H, Owais A. Adherence to guidelines of antibiotic prophylactic use in surgery: a prospective cohort study in North West Bank, Palestine. BMC Surg. 2014;14:69. https://doi.org/10.1186/1471-2482-14-69 Epub 2014/09/11. PubMed PMID: 25204205; PubMed Central PMCID: PMCP MC4168988.

17. Abdel-Aziz A, El-Menyar A, Al-Thani H, Zarour A, Parchani A, Asim M, et al. Adherence of surgeons to antimicrobial prophylaxis guidelines in a tertiary general hospital in a rapidly developing country. Adv Pharmacol Sci. 2013; 2013. https://doi.org/10.1155/2013/842593.

18. Alemkere $\mathrm{G}$. Antibiotic usage in surgical prophylaxis: a prospective observational study in the surgical ward of Nekemte referral hospital. PLoS One. 2018;13(9):e0203523. https://doi.org/10.1371/journal.pone.0203523 Epub 2018/09/14. PubMed PMID: 30212477; PubMed Central PMCID: PMCP MC6136737.

19. Bull AL, Russo PL, Friedman ND, Bennett NJ, Boardman CJ, Richards MJ. Compliance with surgical antibiotic prophylaxis - reporting from a statewide surveillance programme in Victoria, Australia. J Hosp Infect. 2006;63(2):1407. https://doi.org/10.1016/j.jhin.2006.01.018.

20. Al-Momany NH, Al-Bakri AG, Makahleh ZM, Wazaify MM. Adherence to international antimicrobial prophylaxis guidelines in cardiac surgery: a Jordanian study demonstrates need for quality improvement. J Manag Care Pharm. 2009;15(3):262-71. https://doi.org/10.18553/jmcp.2009.15.3.262 Epub 2009/03/31. PubMed PMID: 19326957.

21. Friedman ND, Styles K, Gray AM, Low J, Athan E. Compliance with surgical antibiotic prophylaxis at an Australian teaching hospital. Am J Infect Control. 2013;41(1):71-4. https://doi.org/10.1016/j.ajic.2012.02.012.

22. Grimshaw JM, Eccles MP, Lavis JN, Hill SJ, Squires JE. Knowledge translation of research findings. Implement Sci. 2012;7:50. https://doi.org/10.1186/17485908-7-50 Epub 2012/06/02. PubMed PMID: 22651257; PubMed Central PMCID: PMCPMC3462671.

23. Morris ZS, Wooding S, Grant J. The answer is 17 years, what is the question: understanding time lags in translational research. J R Soc Med. 2011;104(12): 510-20. https://doi.org/10.1258/jrsm.2011.110180 PubMed PMID: 22179294.

24. Grant J, Green L, Mason B. Basic research and health: a reassessment of the scientific basis for the support of biomedical science. Res Eval. 2003;12(3): 217-24. https://doi.org/10.3152/147154403781776618.
25. Gurses AP, Marsteller JA, Ozok AA, Xiao Y, Owens S, Pronovost PJ. Using an interdisciplinary approach to identify factors that affect clinicians' compliance with evidence-based guidelines. Crit Care Med. 2010;38(8 Suppl):S282-91. https://doi.org/10.1097/CCM.0b013e3181e69e02 Epub 2010/ 08/05. PubMed PMID: 20647785.

26. Sheldon TA, Cullum N, Dawson D, Lankshear A, Lowson K, Watt I, et al. What's the evidence that NICE guidance has been implemented? Results from a national evaluation using time series analysis, audit of patients' notes, and interviews. BMJ. 2004;329(7473):999. https://doi.org/10.1136/bmj. 329.7473.999 PubMed PMID: 15514342.

27. Grol R. Successes and failures in the implementation of evidence-based guidelines for clinical practice. Med Care. 2001;39(8 Suppl 2):li46-54. https:// doi.org/10.1097/00005650-200108002-00003 Epub 2001/10/05. PubMed PMID: 11583121

28. Fischer F, Lange K, Klose K, Greiner W, Kraemer A. Barriers and strategies in guideline implementation-a scoping review. Healthcare (Basel). 2016:4(3):36. https://doi.org/10.3390/healthcare4030036 PubMed PMID: 27417624.

29. Liang L, Bernhardsson S, Vernooij RWM, Armstrong MJ, Bussières A, Brouwers MC, et al. Use of theory to plan or evaluate guideline implementation among physicians: a scoping review. Implement Sci. 2017; 12(1):26. https://doi.org/10.1186/s13012-017-0557-0 PubMed PMID: 28241771.

30. Cane J, O'Connor D, Michie S. Validation of the theoretical domains framework for use in behaviour change and implementation research. Implement Sci. 2012;7(1):37. https://doi.org/10.1186/1748-5908-7-37.

31. Atkins L, Francis J, Islam R, O'Connor D, Patey A, Ivers N, et al. A guide to using the Theoretical Domains Framework of behaviour change to investigate implementation problems. Implement Sci. 2017;12(1):77. https:// doi.org/10.1186/s13012-017-0605-9.

32. Moher D, Liberati A, Tetzlaff J, Altman DG, The PG. Preferred Reporting Items for Systematic Reviews and Meta-Analyses: the PRISMA statement. PLoS Med. 2009;6(7):e1000097. https://doi.org/10.1371/journal.pmed. 1000097.

33. Plüddemann A, Aronson JK, Onakpoya I, Heneghan C, Mahtani KR. Redefining rapid reviews: a flexible framework for restricted systematic reviews. BMJ Evid Based Med. 2018;23(6):201. https://doi.org/10.1136/ bmjebm-2018-110990.

34. Hong QN, Fàbregues S, Bartlett G, Boardman F, Cargo M, Dagenais P, et al. The Mixed Methods Appraisal Tool (MMAT) version 2018 for information professionals and researchers. Educ Inf. 2018;34:285-91. https://doi.org/10. 3233/EFI-180221.

35. Al-Azzam SI, Alzoubi KH, Mhaidat NM, Haddadin RD, Masadeh MM, Tumah $H N$, et al. Preoperative antibiotic prophylaxis practice and guideline adherence in Jordan: a multi-centre study in Jordanian hospitals. J Infect Dev Ctries. 2012;6(10):715-20. https://doi.org/10.3855/jidc.1676.

36. Bonfait H, Delaunay C, de Thomasson E, Charrois O. Near-miss event assessment in orthopedic surgery: antimicrobial prophylaxis noncompliance. Orthop Traumatol Surg Res. 2010;96(5):493-9. https://doi.org/10.1016/j.otsr. 2010.03.014 Epub 2010/10/23. PubMed PMID: 20965143.

37. Broom J, Broom A, Kirby E, Post JJ. Improvisation versus guideline concordance in surgical antibiotic prophylaxis: a qualitative study. Infection. 2018;46(4):541-8. https://doi.org/10.1007/s15010-018-1156-y.

38. Chen SY, Ceresnak SR, Motonaga KS, Trela A, Hanisch D, Dubin AM. Antibiotic prophylaxis practices in pediatric cardiac implantable electronic device procedures: a survey of the Pediatric And Congenital Electrophysiology Society (PACES). Pediatr Cardiol. 2018;39(6):1129-33. https://doi.org/10.1007/s00246-018-1865-0 Epub 2018/03/23. PubMed PMID: 29564522.

39. Madubueze CC, Umaru H, Alada A. Attitudes of Nigerian orthopaedic surgeons to the use of prophylactic antibiotics. Int Orthop. 2015;39(11): 2161-5. https://doi.org/10.1007/s00264-015-2822-7.

40. Tan JA, Naik VN, Lingard L. Exploring obstacles to proper timing of prophylactic antibiotics for surgical site infections. Qual Saf Health Care. 2006;15(1):32-8. https://doi.org/10.1136/ashc.2004.012534.

41. Brink AJ, Messina AP, Feldman C, Richards GA, van den Bergh D. From guidelines to practice: a pharmacist-driven prospective audit and feedback improvement model for peri-operative antibiotic prophylaxis in 34 South African hospitals. J Antimicrob Chemother. 2017;72(4):1227-34. https://doi. org/10.1093/jac/dkw523 Epub 2016/12/22. PubMed PMID: 27999061.

42. Bryson DJ, Gulihar A, Aujla RS, Taylor GJS. The hip fracture best practice tariff: early surgery and the implications for MRSA screening and antibiotic 
prophylaxis. Eur J Orthop Surg Traumatol. 2015;25(1):123-7. https://doi.org/ 10.1007/s00590-014-1448-6.

43. Cameron M, Jones $\mathrm{S}$, Adedeji $\mathrm{O}$. Antibiotic prophylaxis audit and questionnaire study: Traffic Light Poster improves adherence to protocol in gastrointestinal surgery. Int J Surg. 2015;19:112-5. https://doi.org/10.1016/j. ijsu.2015.05.008.

44. Carlès M, Gindre S, Aknouch N, Goubaux B, Mousnier A, Raucoules-Aimé M. Improvement of surgical antibiotic prophylaxis: a prospective evaluation of personalized antibiotic kits. J Hosp Infect. 2006;62(3):372-5. https://doi.org/ 10.1016/j.jhin.2005.09.004.

45. Caruso TJ, Wang E, Schwenk HT, Scheinker D, Yeverino C, Tweedy M, et al. A quality improvement initiative to optimize dosing of surgical antimicrobial prophylaxis. Paediatr Anaesth. 2017;27(7):702-10. https://doi. org/10.1111/pan.13137.

46. Collier PE, Rudolph M, Ruckert D, Osella T, Collier NA, Ferrero M. Are preoperative antibiotics administered preoperatively? Am J Med Qual. 1998; 13(2):94-7. https://doi.org/10.1177/106286069801300208.

47. Conaty O, Gaughan L, Downey C, Carolan N, Brophy MJ, Kavanagh R, et al. An interdisciplinary approach to improve surgical antimicrobial prophylaxis. Int J Health Care Qual Assur. 2018;31(2):162-72. https://doi.org/10.1108/ ijhcqa-05-2017-0078 Epub 2018/03/06. PubMed PMID: 29504869.

48. De Almeida SM, Marra AR, Wey SB, Victor EDS, Dos Santos OFP, Edmond MB. Implementation of an antibiotic prophylaxis protocol in an intensive care unit. Am J Infect Control. 2012;40(8):721-5. https://doi.org/10.1016/j.ajic. 2011.09.018.

49. Dimopoulou A, Kourlaba G, Psarris A, Coffin S, Spoulou V, Zaoutis T. Perioperative antimicrobial prophylaxis in pediatric patients in Greece: compliance with guidelines and impact of an educational intervention. J Pediatr Surg. 2016;51(8):1307-11. https://doi.org/10.1016/j.jpedsurg.2015.11.017.

50. Garcell HG, Arias AV, Sandoval CP, Valle Gamboa ME, Sado AB, Alfonso Serrano RN. Impact of a focused antimicrobial stewardship program in adherence to antibiotic prophylaxis and antimicrobial consumption in appendectomies. J Infect Public Health. 2017;10(4):415-20. https://doi.org/ 10.1016/j.jiph.2016.06.006.

51. Haynes K, Linkin DR, Fishman NO, Bilker WB, Strom BL, Pifer EA, et al. Effectiveness of an information technology intervention to improve prophylactic antibacterial use in the postoperative period. J Am Med Inform Assoc. 2011;18(2):164-8. https://doi.org/10.1136/jamia.2009.002998.

52. Hermsen ED, Shull SS, Puumala SE, Rupp ME. Improvement in prescribing habits and economic outcomes associated with the introduction of a standardized approach for surgical antimicrobial prophylaxis. Infect Control Hosp Epidemiol. 2008;29(5):457-61. https://doi.org/10.1086/587811.

53. Hincker A, Ben Abdallah A, Avidan M, Candelario P, Helsten D. Electronic medical record interventions and recurrent perioperative antibiotic administration: a before-and-after study. Can J Anaesth. 2017;64(7):716-23. https:/doi.org/10.1007/ s12630-017-0885-1 Epub 2017/04/20. PubMed PMID: 28421546.

54. Kao LS, Lew DF, Doyle PD, Carrick MM, Jordan VS, Thomas EJ, et al. A tale of 2 hospitals: a staggered cohort study of targeted interventions to improve compliance with antibiotic prophylaxis guidelines. Surgery. 2010;148(2):25562. https://doi.org/10.1016/j.surg.2010.04.003 Epub 2010/05/25. PubMed PMID: 20494387.

55. Kim ES, Park SW, Lee CS, Gyung Kwak Y, Moon C, Kim BN. Impact of a national hospital evaluation program using clinical performance indicators on the use of surgical antibiotic prophylaxis in Korea. Int J Infect Dis. 2012; 16(3):e187-e92. https://doi.org/10.1016/j.jijd.2011.11.010.

56. Kritchevsky SB, Braun BI, Bush AJ, Bozikis MR, Kusek L, Burke JP, et al. The effect of a quality improvement collaborative to improve antimicrobial prophylaxis in surgical patients: a randomized trial. Ann Intern Med. 2008; 149(7):472-80. https://doi.org/10.7326/0003-4819-149-7-200810070-00007 w89-93. Epub 2008/10/08. PubMed PMID: 18838727.

57. Lingard L, Regehr G, Cartmill C, Orser B, Espin S, Bohnen J, et al. Evaluation of a preoperative team briefing: a new communication routine results in improved clinical practice. BMJ Qual Saf. 2011;20(6):475-82. https://doi.org/ 10.1136/bmjgs.2009.032326 Epub 2011/02/10. PubMed PMID: 21303767.

58. Nair BG, Newman SF, Peterson GN, Wu WY, Schwid HA. Feedback mechanisms including real-time electronic alerts to achieve near 100\% timely prophylactic antibiotic administration in surgical cases. Anesth Analg. 2010;111(5):1293-300. https://doi.org/10.1213/ANE.0b013e3181f46d89 Epub 2010/09/16. PubMed PMID: 20841414

59. Nair BG, Newman SF, Peterson GN, Schwid HA. Automated electronic reminders to improve redosing of antibiotics during surgical cases: comparison of two approaches. Surg Infect (Larchmt). 2011;12(1):57-63. https://doi.org/10.1089/sur.2010.047.

60. O'Reilly M, Talsma A, VanRiper S, Kheterpal S, Burney R. An anesthesia information system designed to provide physician-specific feedback improves timely administration of prophylactic antibiotics. Anesth Analg. 2006;103(4):908-12. https://doi.org/10.1213/01.ane.0000237272.77090.a2 Epub 2006/09/27. PubMed PMID: 17000802.

61. Ozgun H, Ertugrul BM, Soyder A, Ozturk B, Aydemir M. Peri-operative antibiotic prophylaxis: adherence to guidelines and effects of educational intervention. Int J Surg. 2010;8(2):159-63. https://doi.org/10.1016/j.jisu.2009. 12.005 Epub 2009/12/23. PubMed PMID: 20026001.

62. Parker BM, Henderson JM, Vitagliano S, Nair BG, Petre J, Maurer WG, et al. Six sigma methodology can be used to improve adherence for antibiotic prophylaxis in patients undergoing noncardiac surgery. Anesth Analg. 2007; 104(1):140-6. Epub 2006/12/21. PubMed PMID: 17179259. https://doi.org/10. 1213/01.ane.0000250371.76725.2e.

63. Ribed A, Monje B, García-González X, Sanchez-Somolinos M, Sanz-Ruiz P, Rodríguez-González CG, et al. Improving surgical antibiotic prophylaxis adherence and reducing hospital readmissions: a bundle of interventions including health information technologies. Eur J Hosp Pharm. 2018. https:// doi.org/10.1136/ejhpharm-2018-001666.

64. Riggi G, Castillo M, Fernandez M, Wawrzyniak A, Vigoda M, Eber S, et al. Improving compliance with timely intraoperative redosing of antimicrobials in surgical prophylaxis. Infect Control Hosp Epidemiol. 2014;35(10):1236-40. https://doi.org/10.1086/678058.

65. Ritchie S, Scanlon N, Lewis M, Black PN. Use of a preprinted sticker to improve the prescribing of prophylactic antibiotics for hip fracture surgery. Qual Saf Health Care. 2004;13(5):384-7. https://doi.org/10.1136/qshc.2003. 009696.

66. Rosenberg AD, Wambold D, Kraemer L, Begley-Keyes M, Zuckerman SL, Singh $N$, et al. Ensuring appropriate timing of antimicrobial prophylaxis. J Bone Joint Surg. 2008;90(2):226-32. https://doi.org/10.2106/JBJS.G.00297.

67. Schwann NM, Bretz KA, Eid S, Burger T, Fry D, Ackler F, et al. Point-of-care electronic prompts: an effective means of increasing compliance, demonstrating quality, and improving outcome. Anesth Analg. 2011;113(4): 869-76. https://doi.org/10.1213/ANE.0b013e318227b511 Epub 2011/07/27. PubMed PMID: 21788319.

68. Shapiro R, Laignel R, Kowcheck C, White V, Hashmi M. Modifying preoperative antibiotic overuse in gynecologic surgery. Int J Health Care Qual Assur. 2018;31(5):400-5. https://doi.org/10.1108/JHCQA-04-2017-0066.

69. Sutherland T, Beloff J, Lightowler M, Liu X, Nascimben L, Kaye AD, et al. Description of a multidisciplinary initiative to improve SCIP measures related to pre-operative antibiotic prophylaxis compliance: a single-center success story. Patient Saf Surg. 2014;8(1). https://doi.org/10.1186/s13037-014-0037-2.

70. Telfah S, Nazer L, Dirani M, Daoud F. Improvement in adherence to surgical antimicrobial prophylaxis guidelines after implementation of a multidisciplinary quality improvement project. Sultan Qaboos Univ Med J. 2015;15(4):e523-e7. https://doi.org/10.18295/squmj.2015.15.04.014 Epub 2015/11/23. PubMed PMID: 26629381

71. Wax DB, Beilin Y, Levin M, Chadha N, Krol M, Reich DL. The effect of an interactive visual reminder in an anesthesia information management system on timeliness of prophylactic antibiotic administration. Anesth Analg. 2007;104(6):1462-6. https://doi.org/10.1213/01.ane.0000263043.56372. $5 f$ table of contents. Epub 2007/05/22. PubMed PMID: 17513642.

72. Whitman G, Cowell V, Parris K, McCullough P, Howard T, Gaughan J, et al. Prophylactic antibiotic use: hardwiring of physician behavior, not education, leads to compliance. J Am Coll Surg. 2008;207(1):88-94. https://doi.org/10. 1016/j.jamcollsurg.2008.01.023.

73. Willems L, Simoens S, Laekeman G. Follow-up of antibiotic prophylaxis: impact on compliance with quideliness and financial outcomes. J Hosp Infect. 2005;60(4):333-9. https://doi.org/10.1016/j.jhin.2004.12.026.

74. Zanetti G, Flanagan HL Jr, Cohn LH, Giardina R, Platt R. Improvement of intraoperative antibiotic prophylaxis in prolonged cardiac surgery by automated alerts in the operating room. Infect Control Hosp Epidemiol. 2003;24(1):13-6. https://doi.org/10.1086/502109.

75. Zanotto AR, Heineck I, Ferreira MB. Antibiotic prophylaxis in cholecystectomies in a teaching hospital in Brazil. Ann Pharmacother. 2006; 40(11):2003-7. https://doi.org/10.1345/aph.1H165.

76. Zhou L, Ma J, Gao J, Chen S, Bao J. Optimizing prophylactic antibiotic practice for cardiothoracic surgery by pharmacists' effects. Medicine (Baltimore). 2016;95(9). https://doi.org/10.1097/MD.0000000000002753. 
77. Broom JK, Broom AF, Kirby ER, Post JJ. How do professional relationships influence surgical antibiotic prophylaxis decision making? A qualitative study. Am J Infect Control. 2018;46(3):311-5. https://doi.org/10.1016/j.ajic. 2017.09.004.

78. Giusti A, Spila Alegiani S, Ciofi Degli Atti ML, Colaceci S, Raschetti R, Arace P, et al. Surgical antibiotic prophylaxis in children: a mixed method study on healthcare professionals attitudes. BMC Pediatr. 2016;16(1):203. https://doi. org/10.1186/s12887-016-0739-y Epub 2016/12/07. PubMed PMID: 27919244; PubMed Central PMCID: PMCPMC5139116.

79. Nobile M, Bronzin S, Navone P, Colombo M, Calori GM, Auxilia F. Reinforcing good practice: implementation of guidelines at hospital G. Pini. Injury. 2014; 45(S6):S2-8. https://doi.org/10.1016/j.injury.2014.10.015.

80. Knox MC, Edye M. Educational antimicrobial stewardship intervention ineffective in changing surgical prophylactic antibiotic prescribing. Surg Infect (Larchmt). 2016;17(2):224-8. https://doi.org/10.1089/sur.2015.194.

81. Nemeth TA, Beilman GJ, Hamlin CL, Chipman JG. Preoperative verification of timely antimicrobial prophylaxis does not improve compliance with guidelines. Surg Infect (Larchmt). 2010;11(4):387-91. https://doi.org/10.1089/ sur.2008.054

82. Putnam LR, Chang CM, Rogers NB, Podolnick JM, Sakhuja S, Matusczcak M, et al. Adherence to surgical antibiotic prophylaxis remains a challenge despite multifaceted interventions. Surgery. 2015;158(2):413-9. https://doi. org/10.1016/j.surg.2015.04.013 Epub 2015/06/10. PubMed PMID: 26054317.

83. Chauveaux D. Preventing surgical-site infections: measures other than antibiotics. Orthop Traumatol Surg Res. 2015;101(1, Supplement):S77-83. https://doi.org/10.1016/j.otsr.2014.07.028.

84. Culleton B. Evidence-based decision-making 4: development and limitations of clinical practice guidelines. Methods Mol Biol. 2015;1281:443-53. https:// doi.org/10.1007/978-1-4939-2428-8_26 Epub 2015/02/20. PubMed PMID: 25694326

85. lerano C, Thursky K, Peel T, Rajkhowa A, Marshall C, Ayton D. Influences on surgical antimicrobial prophylaxis decision making by surgical craft groups, anaesthetists, pharmacists and nurses in public and private hospitals. PLoS One. 2019:14(11):e0225011. https://doi.org/10.1371/journal.pone.0225011 Epub 2019/11/15. PubMed PMID: 31725771; PubMed Central PMCID: PMCP MC6855473.

86. Davis DA, McMahon GT. Translating evidence into practice: lessons for CPD. Med Teach. 2018;40(9):892-5. https://doi.org/10.1080/0142159X.2018.1481285.

87. Tudor Car L, Soong A, Kyaw BM, Chua KL, Low-Beer N, Majeed A. Health professions digital education on clinical practice guidelines: a systematic review by Digital Health Education collaboration. BMC Med. 2019;17(1):139. https://doi.org/10.1186/s12916-019-1370-1.

88. Bero LA, Grilli R, Grimshaw JM, Harvey E, Oxman AD, Thomson MA. Closing the gap between research and practice: an overview of systematic reviews of interventions to promote the implementation of research findings. The Cochrane Effective Practice and Organization of Care Review Group. BMJ. 1998:317(7156):465-8. https://doi.org/10.1136/bmj.317.7156.465 PubMed PMID: 9703533.

89. van Buijtene A, Foster D. Does a hospital culture influence adherence to infection prevention and control and rates of healthcare associated infection? A literature review. J Infect Prev. 2019:20(1):5-17. https://doi.org/ 10.1177/1757177418805833 Epub 2018/11/09. PubMed PMID: 30719083.

90. Braithwaite J, Herkes J, Ludlow K, Lamprell G, Testa L. Association between organisational and workplace cultures, and patient outcomes: systematic review protocol. BMJ Open. 2016;6(12):e013758. https://doi.org/10.1136/ bmjopen-2016-013758 Epub 2016/12/03. PubMed PMID: 27909040; PubMed Central PMCID: PMCPMC5168669.

91. Tsiga E, Panagopoulou E, Sevdalis N, Montgomery A, Benos A. The influence of time pressure on adherence to guidelines in primary care: an experimental study. BMJ Open. 2013;3(4):e002700. https://doi.org/10.1136/ bmjopen-2013-002700.

92. Vehko T, Hyppönen H, Puttonen S, Kujala S, Ketola E, Tuukkanen J, et al. Experienced time pressure and stress: electronic health records usability and information technology competence play a role. BMC Med Inform Decis Mak. 2019;19(1):160. https://doi.org/10.1186/s12911-019-0891-z.

93. O'Daniel M, Rosenstein AH. Professional communication and team collaboration. In: Hughes RG, editor. Patient safety and quality: an evidencebased handbook for nurses. Rockville: Agency for Healthcare Research and Quality (US); 2008.

94. Wilkinson DJ. Anaesthesia. BMJ. 1999;318(7187):S2-7187. https://doi.org/10. 1136/bmj.318.7187.2
95. Kirkland KB, Briggs JP, Trivette SL, Wilkinson WE, Sexton DJ. The impact of surgical-site infections in the 1990s: attributable mortality, excess length of hospitalization, and extra costs. Infect Control Hosp Epidemiol. 1999;20(11): 725-30. https://doi.org/10.1086/501572 Epub 1999/12/02. PubMed PMID: 10580621.

96. Ivers N, Jamtvedt G, Flottorp S, Young JM, Odgaard-Jensen J, French SD, et al. Audit and feedback: effects on professional practice and healthcare outcomes. Cochrane Database Syst Rev. 2012;6. https://doi.org/10.1002/ 14651858.CD000259.pub3 PubMed PMID: CD000259.

97. Forsner T, Hansson J, Brommels M, Wistedt AA, Forsell Y. Implementing clinical guidelines in psychiatry: a qualitative study of perceived facilitators and barriers. BMC Psychiatry. 2010;10:8. https://doi.org/10.1186/1471-244X10-8 PubMed PMID: 20089141

98. Cabana MD, Rushton JL, Rush AJ. Implementing practice guidelines for depression: applying a new framework to an old problem. Gen Hosp Psychiatry. 2002;24(1):35-42. https://doi.org/10.1016/s0163-8343(01)00169-4 Epub 2002/01/30. PubMed PMID: 11814532.

99. Grimshaw JM, Eccles MP. Is evidence-based implementation of evidencebased care possible? Med J Aust. 2004;180(S6):S50-1 Epub 2004/03/12. PubMed PMID: 15012580.

100. Grimshaw J, Thomas R, Maclennan G, Fraser C, Ramsay C, Vale L, et al. Effectiveness and efficiency of guideline dissemination and implementation strategies. Health Technol Assess. 2004;8(6). https://doi.org/10.3310/hta8060.

101. McKenzie JE, Brennan SE. Chapter 12: Synthesizing and presenting findings using other methods. In: Higgins JPT, Thomas J, Chandler J, Cumpston M, Li T, Page MJ, Welch VA (editors). Cochrane Handbook for Systematic Reviews of Interventions version 6.1 (updated September 2020). Cochrane. 2020. Available from http://www.training.cochrane.org/handbook.

102. Ng RS, Chong CP. Surgeons' adherence to guidelines for surgical antimicrobial prophylaxis - a review. Australas Med J. 2012;5(10):534-40. https://doi.org/10.4066/amj.2012.1312 Epub 2012/11/23. PubMed PMID: 23173017; PubMed Central PMCID: PMCPMC3494825.

103. van Kasteren ME, Kullberg BJ, de Boer AS, Mintjes-de Groot J, Gyssens IC Adherence to local hospital guidelines for surgical antimicrobial prophylaxis: a multicentre audit in Dutch hospitals. J Antimicrob Chemother. 2003;51(6): 1389-96. https://doi.org/10.1093/jac/dkg264 Epub 2003/05/15. PubMed PMID: 12746377.

104. Gouvêa M, Novaes CO, Pereira DMT, Iglesias AC. Adherence to guidelines for surgical antibiotic prophylaxis: a review. Braz J Infect Dis. 2015;19(5):51724. https://doi.org/10.1016/j.bjid.2015.06.004.

\section{Publisher's Note}

Springer Nature remains neutral with regard to jurisdictional claims in published maps and institutional affiliations.

Ready to submit your research? Choose BMC and benefit from

- fast, convenient online submission

- thorough peer review by experienced researchers in your field

- rapid publication on acceptance

- support for research data, including large and complex data types

- gold Open Access which fosters wider collaboration and increased citations

- maximum visibility for your research: over $100 \mathrm{M}$ website views per year

At BMC, research is always in progress.

Learn more biomedcentral.com/submissions 